\title{
Large- $Q^{2}$ behavior of the pion electromagnetic form factor*
}

\author{
Marvin L. Goldberger and Davison E. Soper \\ Joseph Henry Laboratories, Princeton University, Princeton, New Jersey 08540
}

Alan H. Guth

Department of Physics, Columbia University, New York, New York 10027

(Received 22 March 1976)

\begin{abstract}
We study the large- $Q^{2}$ behavior of the electromagnetic form factor of the pion, which is viewed as a quarkantiquark bound state in a (nongauge) quantum field theory. When the pion's Bethe-Salpeter wave function is expanded in O(4) partial waves, it is found that the information needed about the partial-wave amplitudes is their scaling behavior at large momentum and the locations of their poles in the complex $J$ plane. This information is determined by using the operator-product expansion, conformal invariance at short distances, and a regularity property that holds at least in the ladder model. The resulting behavior of the form factor is roughly $F\left(Q^{2}\right) \sim\left(Q^{2}\right)^{-1}$, with corrections due to anomalous dimensions.
\end{abstract}

\section{INTRODUCTION}

The dependence of electromagnetic form factors on the squared four-momentum transfer $Q^{2}$ as $Q^{2} \rightarrow \infty$ has been the subject of much theoretical study. It is well known that for a spin-0 particle, described say in a $\phi^{3}$-type theory, in lowest-order perturbation theory, the form factor $F\left(Q^{2}\right) \sim\left(Q^{2}\right)^{-1}$ as $Q^{2} \rightarrow \infty$. It was pointed out by a number of authors, ${ }^{1}$ that if the particle were regarded as composite (for example, if the spin-0 particle in question were the bound state of two spin-0 particles), at least within the framework of a ladder approximation to the Bethe-Salpeter equation, then $F\left(Q^{2}\right)$ $\sim\left(Q^{2}\right)^{-2}$ as $Q^{2} \rightarrow \infty$. It was recognized by Ciafaloni and $\mathrm{Menotti}^{2}$ that the precise behavior depended on the spin of the constituents making up the bound states and possibly on how singular the interaction between constituents was.

More recently, attempts have been made to study the asymptotic behavior of form factors in a more model-independent manner using the notion of conformal invariance as a guide. ${ }^{3,4}$ These investigations have given a variety of answers.

In the present paper we study the problem of the electromagnetic form factor of a pseudoscalar particle of mass $M$ (called a pion) composed of two spin $-\frac{1}{2}$ constituents (quarks). The principal contribution to the form factor comes from what is called the disconnected graph [see Fig. 2(a)], where the pion disintegrates virtually into its spin- $\frac{1}{2}$ constituents, one of which absorbs the large momentum transfer from the electromagnetic current and then re-forms with the unstruck quark to reconstitute the final pion. The fundamental ingredients needed for the evaluation then are the pion wave function, the propagator for the quarks, and their electromagnetic vertex.

To analyze these components of the computation we introduce an $\mathrm{O}(4)$ expansion of the pion wave function in Sec. II and discuss the extension of the "partial-wave amplitudes" to complex angular momenta. We utilize this expansion to carry out part of the loop integration implied by Fig. 2(a) (Sec. III). The remaining computation then leans heavily on an analysis of the operator-product expansion for two fermion fields and the restrictions imposed on this expansion by conformal invariance (Sec. IV). It is necessary to assume that the behavior of the wave function when one quark is far off the mass shell and the other quark has fixed $q^{2}$ is the same as that obtained from conformal invariance, although conformal invariance is strictly applicable only when $q^{2}$ is much larger than any mass squared. The final evaluation of the form factor is given in Sec. V and a summary of our results together with a comparison with those of others is given in Sec. VI. In an appendix the intricacies associated with the quark propagator and electromagnetic vertex modifications are treated in some detail. We refer occasionally to results obtained from a ladder-model calculation which will be published elsewhere. ${ }^{5}$

\section{THE PION WAVE FUNCTION \\ Definition and symmetries}

We begin with the definition and symmetry properties of the Bethe-Salpeter wave function of the pion bound state.

Our notation is as follows: The metric tensor is $g_{\mu \nu}=\operatorname{diag}(-1,1,1,1) ; \gamma$ matrices obey $\left\{\gamma_{\mu}, \gamma_{\nu}\right\}=2 g_{\mu \nu}$, $\gamma^{\mu \dagger}=g_{\mu \nu} \gamma^{\nu}$ for $\mu=0,1,2,3 ; \gamma^{4}=+i \gamma^{0}, \bar{\psi}=\psi^{\dagger} \gamma^{4} ; \not P$ $=\gamma^{\mu} g_{\mu \nu} P^{\nu} ; \gamma_{5}=\gamma^{1} \gamma^{2} \gamma^{3} \gamma^{4}$; states are covariantly 
normalized to $\left\langle P \mid P^{\prime}\right\rangle=(2 \pi)^{3} 2 E \delta\left(\overrightarrow{\mathrm{P}}^{\prime}-\overrightarrow{\mathrm{P}}\right)$.

We consider the pion to be a bound state of mass $M$ of a spin- $\frac{1}{2}$ quark of type $A$ and a spin- $-\frac{1}{2}$ antiquark of type $B$. [The $\mathrm{SU}(3)$ and color symmetry properties of the quark are suppressed.] The Bethe-Salpeter wave function of the pion is then (see Fig. 1)

$\chi_{\alpha \beta}\left(q^{\nu}+P^{\nu}, q^{\nu}\right)=\int d x e^{i q^{\cdot x}}\left\langle 0\left|T\left\{\psi_{\alpha}^{(A)}(0) \bar{\psi}_{B}^{(B)}(x)\right\}\right| P^{\nu}\right\rangle$.

This wave function can be continued off the pion mass shell, $P^{2}=-M^{2}$, by defining

$$
\begin{aligned}
\chi_{\alpha \beta}\left(q^{\nu}+\right. & \left.P^{\nu}, q^{\nu}\right) \\
=\frac{1}{G_{\phi}\left(P^{2}\right)} \int & d^{4} x d^{4} y e^{i(q \cdot x+P \cdot y)} \\
& \quad \times\left\langle 0\left|T\left\{\psi_{\alpha}^{(A)}(0) \bar{\psi}_{B}^{(B)}(x) \phi^{\dagger}(y)\right\}\right| 0\right\rangle,
\end{aligned}
$$

where $\phi(x)$ is any convenient pion interpolating field [normalized so that $\left.\left\langle 0|\phi(x)| P^{\nu}\right\rangle=e^{i P \cdot x}\right]$ and $G_{\phi}\left(P^{2}\right)$ is the corresponding pion propagator.

One can also define an off-shell wave function appropriate for a final-state pion by (see Fig. 1)

$$
\begin{aligned}
\bar{\chi}_{\boldsymbol{\alpha} \beta}\left(q^{\nu}, q^{\nu}+P^{\nu}\right) \\
=\frac{1}{G_{\phi}\left(P^{2}\right)} \int d x d y e^{-i(q \cdot x+P \cdot y)} \\
\times\left\langle 0\left|T\left\{\phi(y) \psi_{\boldsymbol{\alpha}}^{(B)}(x) \bar{\psi}_{\beta}^{(A)}(0)\right\}\right| 0\right\rangle .
\end{aligned}
$$

When $P^{2}=-M^{2}, P^{0}>0$ this is

$\bar{\chi}_{\boldsymbol{\alpha} \beta}\left(q^{\nu}, q^{\nu}+P^{\nu}\right)=\int d x e^{-i q \cdot x}\left\langle P^{\nu}\left|T\left\{\psi_{\boldsymbol{\alpha}}^{(B)}(x) \bar{\psi}_{\boldsymbol{\beta}}^{(\boldsymbol{A})}(0)\right\}\right| 0\right\rangle$.

The wave functions $\chi$ and $\bar{\chi}$ are related by $P T$
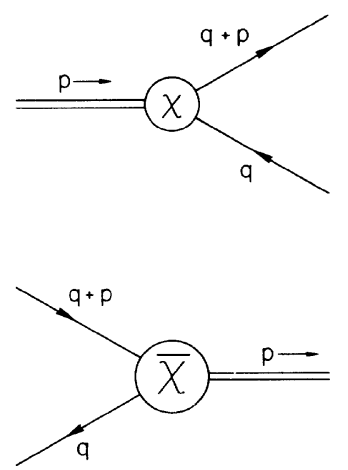

FIG. 1. The Bethe-Salpeter wave functions $\chi$ and $\bar{\chi}$. invariance:

$\chi_{\beta \alpha}\left(q^{\nu}+P^{\nu}, q^{\nu}\right)=-T_{\alpha \alpha^{\prime}} \bar{\chi}_{\alpha^{\prime} \beta},\left(q^{\nu}, q^{\nu}+P^{\nu}\right) T_{\beta^{\prime} \beta}^{-1}$,

where $T=i \gamma^{1} \gamma^{3}$ is the usual time-reversal matrix with $T \gamma_{\mu} T^{-1}=\gamma_{\mu}{ }^{T}$. The factor -1 here is the pion parity.

\section{O(4) expansion}

The wave function $\chi\left(q^{\nu}+P^{\nu}, q^{\nu}\right)$ is an analytic function of the complex 4-vectors $q^{\nu}$ and $P^{\nu}$ (in a certain region of $q^{\nu}, P^{\nu}$ ). We will be largely concerned with $\chi$ evaluated at Euclidean points, where $q^{1}, q^{2}, q^{3}, P^{1}, P^{2}, P^{3}$ are real and $q^{0}$, and $P^{0}$ are imaginary. At such points $\chi$ transforms simply under the roation group in four dimensions, $\mathrm{O}(4)$, which rotates, for example, the vectors $\left(q^{1}, q^{2}, q^{3}\right.$, $\left.i q^{0}\right)$. We will make use of the expansion of $\chi$ in representation functions of $\mathrm{O}(4)$. The basis functions that we choose are

$$
\begin{aligned}
& G_{J}^{1}\left(q^{\nu}, P^{\nu}\right)=i \gamma_{5}(-2)^{J} q^{-J}\left\{q^{\mu_{1}} \cdots q^{\mu_{J}}\right\}\left\{P_{\mu_{1}} \cdots P_{\mu_{J}}\right\} \\
& =i \gamma_{5}(-1)^{J} P^{J} U_{J}(\hat{P} \cdot \hat{q}) \text {, } \\
& G_{J}^{2}\left(q^{\nu}, P^{\nu}\right)=i\left[\gamma_{\alpha}, \gamma^{\beta}\right] \gamma_{5}(-2)^{J-1} q^{-J} \\
& \times\left\{q^{\mu_{1}} \cdots q^{\mu_{J-1}} q^{\alpha}\right\}\left\{P_{\mu_{1}} \cdots P_{\mu_{J-1}} P_{\beta}\right\} \\
& =i[\hat{P}, \hat{q}] \gamma_{5} \frac{(-1)^{J}}{2 J} P^{J} U_{J}^{\prime}(\hat{P} \cdot \hat{q}) \text {, } \\
& G_{J}^{3}\left(q^{\nu}, P^{\nu}\right)=\gamma^{\alpha} \gamma_{5}(-2)^{J} q^{-(J-1)}\left\{q^{\mu_{1}} \cdots q^{\mu_{J-1}}\right\} \\
& \times\left\{P_{\mu_{1}} \cdots P_{\mu_{J-1}} P_{\alpha}\right\} \\
& =\frac{(-1)^{J}}{J} P^{J}\left[\hat{P} \gamma_{5} U_{J}^{\prime}(\hat{P} \cdot \hat{q})-\tilde{q} \gamma_{5} U_{J-1}^{\prime}(\hat{P} \cdot \hat{q})\right] \text {, } \\
& G_{J}^{4}\left(q^{\nu}, P^{\nu}\right)=\gamma_{\alpha} \gamma_{5}(-2)^{J+1} q^{-(J+1)}\left\{q^{\mu_{1}} \cdots q^{\mu_{J}} q^{\alpha}\right\} \\
& \times\left\{P_{\mu_{1}} \cdots P_{\mu_{J}}\right\} \\
& =-\frac{(-1)^{J}}{J+1} P^{J}\left[\hat{q} \gamma_{5} U_{J+1}^{\prime}(\hat{P} \cdot \hat{q})-\hat{p} \gamma_{5} U_{J}^{\prime}(\hat{P} \cdot \hat{q})\right] \text {. }
\end{aligned}
$$

Here \{\} indicates that the tensor inside is to be made traceless and symmetric. The function $U_{J}(\cos \theta)$ is the Chebyshev polynomial of the second kind, ${ }^{6}$

$$
U_{J}(\cos \theta)=\frac{\sin [(J+1) \theta]}{\sin \theta},
$$

and $U_{J}^{\prime}=d U_{J} / d \cos \theta$. We have defined $P=\left(P^{\nu} P_{\nu}\right)^{1 / 2}$, $\hat{P}^{\nu}=P^{\nu} / P$, and similarly for $q, \hat{q}^{\nu}$.

These $O(4)$ basis functions and the traceless symmetric operation \{\} are discussed more fully in Ref. 7. There it is also shown that these functions $G_{J}^{i}$ are a complete set of functions for the expansion of a wave function of a pseudoscalar particle evaluated at Euclidean momenta. Thus we can write 


$$
\chi\left(q^{\nu}+P^{\nu}, q^{\nu}\right)=\sum_{J=0}^{\infty} \sum_{i=1}^{4} \chi_{J}^{i}\left(q^{2}, P^{2}\right) G_{J}^{i}\left(q^{\nu}, P^{\nu}\right) .
$$

(Actually the sum for $i=2$ and 3 should begin at $J=1$; we ensure this by taking $\chi_{0}^{2}=\chi_{0}^{3}=0$.)

We also expand $\bar{\chi}\left(q^{\nu}+P^{\nu}, q^{\nu}\right)$ using the relation (2.5):

$\bar{\chi}\left(q^{\nu}, q^{\nu}+P^{\nu}\right)=-\sum_{J=0}^{\infty} \sum_{i=1}^{4} \chi_{J}^{i}\left(q^{2}, P^{2}\right) T^{-1} G_{J}^{i}\left(q^{\nu}, P^{\nu}\right)^{T} T$.

[The factors $P^{J}$ in the definition of the basis functions have been chosen so that the partial-wave amplitudes $\chi_{J}^{i}\left(q^{2}, P^{2}\right)$ will not have a branch point at $P^{2}=0$, which would introduce complications into the analytic continuation from Euclidean values of $P^{2}$ back to $P^{2}=-M^{2}$. The factors of $i$ have been chosen so that the $\chi_{J}^{i}$ will be real at $P^{2}=-M^{2}{ }^{8}$ The factors $(-1)^{J}$ have been chosen so that when the $\chi_{J}^{i}$ are analytically continued into the complex $J$ plane as discussed below they will be well behaved as $|\operatorname{Im} J| \rightarrow \infty$. Finally, the overall normalizations of the $G_{J}^{i}$ are essentially arbitrary.]

\section{The complex $J$ plane}

The $\mathrm{O}(4)$ expansion of the Bethe-Salpeter wave function that we have used has, of course, been defined for integer values of the "angular momentum" $J$. Furthermore, we have assumed that the angle $\theta$ defined by $\cos \theta=\hat{q} \cdot \hat{P}$ is real. The expansion converges inside an ellipse in the complex $\cos \theta$ plane which has foci at \pm 1 . This result is strictly analogous to the more familiar $\mathrm{O}(3) \mathrm{ex}-$ pansion of a scattering amplitude in terms of Legendre polynomials. As in that case, the size of the ellipse is dictated by the location of the nearest singularity in the $\cos \theta$ plane. For our application to form factors we shall require the behavior of the wave function $\chi(P+q, q)$ in a regime where $P \cdot q$ $\rightarrow \infty$, and as a result the $O(4)$ expansion as it stands is not very useful. Furthermore, as a purely mathematical technique, to evaluate the form factor we shall find it very useful to make a Sommerfeld-Watson transformation and then will require the "partial-wave amplitudes" $\chi_{J}^{i}\left(q^{2}\right)$ for complex $J$ values. We turn, therefore, to an appropriate definition of the $\chi_{J}^{i}\left(q^{2}\right)$ for complex $J$.

We begin by studying what we have called $\chi_{J}^{1}$, which is defined by

$$
P^{J} \chi_{J}^{1} \equiv g_{J}^{1}=\frac{(-1)^{J}}{2 \pi^{2} i} \int d \Omega_{q}\left[\frac{1}{4} \operatorname{Tr}\left(\gamma_{5} \chi\right)\right] U_{J}(\hat{P} \cdot \hat{q}),
$$

where we have used the fact that

$$
G_{J}^{1}=i(-P)^{J} U_{J}(\cos \theta) \gamma_{5}, \quad U_{J}(\cos \theta)=\frac{\sin (J+1) \theta}{\sin \theta}
$$

together with the orthogonality relation

$$
\int d \Omega U_{J}(\cos \theta) U_{L}(\cos \theta)=2 \pi^{2} \delta_{J, L} \quad(J, L \geqslant 0) .
$$

Later we shall give the definitions of the other $\chi_{J}^{i}\left(q^{2}\right)$, but for now we shall concentrate on $g_{J}^{1}\left(q^{2}\right)$, which we write simply as $g_{J}$, suppressing the $q^{2}$ dependence. Writing $\frac{1}{4} \operatorname{Tr}\left(-i \gamma_{5} \chi\right)$ as $F(\cos \theta)$, we can see immediately that the defining integral

$$
g_{J}=\frac{(-1)^{J}}{2 \pi^{2}} \int d \Omega F(\cos \theta) U_{J}(\cos \theta)
$$

does not provide a suitable continuation for complex $J$. Aside from the factor of $(-1)^{J}$, which will turn out to be useful, the real problem comes from the $U_{J}(\cos \theta)$, which leads to a $g_{J}$ which grows exponentially for large positive or negative imaginary $J$. This behavior would prevent a useful Sommerfeld-Watson transformation. This behavior is quite analogous to the fact that the familiar $\mathrm{O}(3)$ partial-wave amplitude $f_{l}$ defined by

$$
f_{l}=\frac{1}{2} \int_{-1}^{+1} d(\cos \theta) P_{l}(\cos \theta) F(\cos \theta)
$$

is not appropriate for extension into the complex $l$ plane $\left(P_{l}\right.$ is a Legendre polynomial, and $F$ is the scattering amplitude).

Now we recall that we obtain a good analytic continuation by using the fact that $F$ has a dispersion representation

$$
F(\cos \theta)=\int_{1}^{\infty} \frac{d z}{\pi} \frac{A(z)}{z-\cos \theta} .
$$

Substituting this into the definition of $f_{l}$, we find first for integer $l$

$$
f_{l}=\int_{1}^{\infty} \frac{d z}{\pi} A(z) Q_{l}(z),
$$

where $Q_{l}(z)$ is a Legendre function of the second kind. This so-called Froissart-Gribov definition of a partial-wave amplitude provides a definition of $f_{l}$ for complex $l$ provided $\operatorname{Re} l$ is sufficiently large. We follow precisely the same procedure for the $O(4)$ expansion.

We must first write a dispersion relation that expresses the dependence of the Bethe-Salpeter wave function $\chi(P+q, q)$ on the variable $(P+q)^{2}$. To derive the desired representation in the case $P^{2}=-M^{2}$, return to the definition of $\chi$ as a timeordered product and remark that for $q^{2}$ spacelike we may write it as

$$
\chi(P+q, q)=\int d^{4} x e^{i q \cdot x}\left\langle 0\left|\{\psi(0), \bar{\psi}(x)\} \theta\left(-x_{0}\right)\right| P\right\rangle
$$


since

$$
T(\psi(0) \bar{\psi}(x))=\{\psi(0), \bar{\psi}(x)\} \theta\left(-x_{0}\right)-\bar{\psi}(x) \psi(0),
$$

and the last term vanishes when the integration over $\chi$ is carried out. In a frame in which $\overrightarrow{\mathrm{p}} \cdot \overrightarrow{\mathrm{q}}=0$ we may write

$$
\exp (i q \cdot x)=\exp \left[-i q_{0} x_{0}+i\left(q_{0}^{2}+q^{2}\right)^{1 / 2} \hat{n} \cdot \overrightarrow{\mathrm{x}}\right],
$$

where $\hat{n}$ is a unit vector in the direction of $\overrightarrow{\mathrm{q}}$. Note that we have reverted to the Minkowski world. The causal structure of the anticommutator, the factor of $\theta\left(-x_{0}\right)$, and the fact that with $q^{2}>0$ (spacelike) there are no growing exponentials as $q_{0}$ ranges from $-\infty$ to $\infty$ make it trivial to establish a dispersion in the variable $q_{0}$ or (what is the same) $-q \cdot P .^{9}$ This argument applies for $P^{2}=-M^{2}$, or, by a slight extension, for any $P^{2}$.

We should, of course, fuss with the Dirac indices, but we may obviously imagine expressing $\chi$ as

$$
\begin{aligned}
\chi(P+q, q)= & i \gamma_{5} F_{1}\left((P+q)^{2}, q^{2}, P^{2}\right) \\
& +i[\not P, q] \gamma_{5} F_{2}+\not P \gamma_{5} F_{3}+q \gamma_{5} F_{4}
\end{aligned}
$$

and writing our dispersion relations for the $F_{i}$. We have then for $F_{1}$, which is the only one which enters our definition of $g_{J}^{1}$ (dropping the index 1),

$$
\left.F\left((P+q)^{2}, q^{2}, P^{2}\right)\right)=\frac{1}{\pi} \int_{m^{2}}^{\infty} d \rho^{2} \frac{F_{D}\left(\rho^{2}, q^{2}, P^{2}\right)}{\rho^{2}+(P+q)^{2}-i \epsilon},
$$

where the lower limit corresponds to the mass of one of our "quarks." Finally we return to the Wick-rotated world and the $-i \epsilon$ plays no role.

We now substitute this representation into our definition of the partial-wave amplitude $g_{J}$ and obtain

$$
g_{J}=\frac{(-1)^{J}}{4 \pi^{3} P q} \int_{m^{2}}^{\infty} d \rho^{2} F_{D}\left(\rho^{2}\right) I_{J}(\omega),
$$

where

$$
\begin{gathered}
I_{J}(\omega)=\int d \Omega \frac{U_{J}(\cos \theta)}{\cosh \omega+\cos \theta}, \\
\cosh \omega=\left(\rho^{2}+P^{2}+q^{2}\right) / 2 P q .
\end{gathered}
$$

To evaluate $I_{J}(\omega)$ most simply we recall the generating function for the polynomials $U_{J}(\cos \theta)$, namely

$$
\left(1+\sigma^{2}+2 \sigma \cos \theta\right)^{-1}=\sum_{L=0}^{\infty}(-1)^{L} \sigma^{L} U_{L}(\cos \theta), \quad \sigma<1 .
$$

Writing $\sigma=e^{-\omega}$ we note that

$$
(\cosh \omega+\cos \theta)^{-1}=2 \sum_{L=0}^{\infty}(-1)^{L} \sigma^{L+1} U_{L}(\cos \theta)
$$

so that $I_{J}(\omega)$ becomes

$$
\begin{aligned}
I_{J}(\omega) & =8 \pi \sum_{L=0}^{\infty}(-1)^{L} \sigma^{L+1} \int_{0}^{\pi} d \theta \sin ^{2} \theta U_{J}(\cos \theta) U_{L}(\cos \theta) \\
& =8 \pi \sum_{L=0}^{\infty}(-1)^{L} \sigma^{L+1} \int_{0}^{\pi} d \theta \sin (J+1) \theta \sin (L+1) \theta \\
& =4 \pi \sum_{L=0}^{\infty}(-1)^{L} \sigma^{L+1} \int_{0}^{\infty} d \theta[\cos (J-L) \theta \\
& =4 \pi^{2} \sum_{L=0}^{\infty}(-1)^{L} \sigma^{L+1}\left(\delta_{L, J}-\delta_{L,-J-2}\right) .
\end{aligned}
$$

Thus

$$
\begin{aligned}
I_{J} & =4 \pi^{2}(-1)^{J} e^{-(J+1) \omega}, \quad J \geqslant 0 \\
& =-4 \pi^{2}(-1)^{J} e^{(J+1) \omega}, \quad J \leqslant-2 .
\end{aligned}
$$

Now, of course, $g_{J}$ was originally defined for positive integer $J$ and we could have instantly used the orthogonality relation for the $U$ 's to obtain the above $J \geqslant 0$ result. Our purpose in giving the tortuous evaluation is that the formal relation

$$
I_{J}(\omega)=-I_{-J-2}(\omega)
$$

suggests the corresponding one for the $g_{J}$ 's. This so-called Lorentz symmetry for the partial-wave amplitudes will, in fact, be established below.

From our $I_{J}(\omega)$ for positive integer $J$ we find for $g_{J}$ the result

$$
g_{J} \equiv P^{J} \chi_{J}^{1}=\frac{1}{\pi P q} \int_{m^{2}}^{\infty} d \rho^{2} e^{-(J+1) \omega} F_{D}\left(\rho^{2}, q^{2}, P^{2}\right),
$$

where, we recall, $\cosh \omega=\left(\rho^{2}+P^{2}+q^{2}\right) / 2 P q$. Notice that as $\rho^{2} \rightarrow \infty$ with $q^{2}$ fixed,

$$
e^{\omega} \sim \frac{\rho^{2}}{P q}\left(1+\frac{q^{2}+P^{2}}{\rho^{2}}+\cdots\right) .
$$

Thus, if $F_{D} \sim a_{1}\left(q^{2}, P^{2}\right)\left(\rho^{2}\right)^{J_{1}}$ as $\rho^{2} \rightarrow \infty$ with $q^{2}$ fixed, then Eq. (2.15) provides a satisfactory definition of $g_{J}$ in the entire half-plane $\operatorname{Re} J>J_{1}$. Notice that this definition makes $g_{J}$ well behaved as $|\operatorname{Im} J| \rightarrow \infty$. Suppose that $F_{D}$ behaves like

$$
\begin{aligned}
a_{1}\left(q^{2}, P^{2}\right)\left(\rho^{2}\right)^{J_{1}}+\cdots+a_{N-1}\left(q^{2}, P^{2}\right)\left(\rho^{2}\right)^{J_{N-1}} & \\
& +f_{R}\left(q^{2}, p^{2}, P^{2}\right),
\end{aligned}
$$

where $J_{1}>J_{2}>\cdots>J_{N}$ and $f_{R}$ falls off faster than $\left(\rho^{2}\right)^{J_{N}}$ as $\rho \rightarrow \infty$. Then the analytically continued partial-wave amplitude $g_{J}$ (or $\chi_{J}^{1}$ ) will be meromorphic in the half-plane $\operatorname{Re} J>J_{N}$, with poles at $J=J_{\alpha}(\alpha=1, \ldots, N-1)$ and at $J=J_{\alpha}-1$, etc. Thus, the locations and residues of poles of the partialwave amplitudes $g_{J}\left(q^{2}\right)$ in the complex $J$ plane provide a direct transcription of the powers $J_{\alpha}$ of $\rho^{2}$ and the corresponding coefficients $a$ that occur in the asymptotic expansion of $F_{D}$ as $\rho^{2} \rightarrow \infty$ with $q^{2}$ fixed. 
We turn now to a discussion of the symmetry relation alluded to above, namely

$$
g_{J}=-g_{-J-2}
$$

for integer $J$. This is quite like the Mandelstam symmetry in the $\mathrm{O}(3)$ case, which is, in fact, a formal consequence of the Froissart-Gribov definition, namely

$$
f_{l}=f_{-l-1}, \quad l=\text { half integer }
$$

since we have the relation

$$
\pi \cot \pi l P_{l}(z)=Q_{l}(z)-Q_{-l-1}(z),
$$

and thus $Q_{l}=Q_{-l-1}$ for half-integer $l$. There does not appear to be a correspondingly simple argument, even of a formal nature, for our situation. We are deeply indebted to Loyal Durand for showing us some unpublished work in collaboration with P. Fishbane and L. Simmons that treats Lorentz symmetry in great detail for scattering amplitudes. We are concerned with a somewhat simpler form of the problem treated by these authors.

We begin by reconsidering the integral we called $I_{J}(\omega)[\mathrm{Eq} .(2.14)]$, but now we allow $J$ to take on arbitrary complex values. We write it now as

$$
\begin{aligned}
\frac{I(J)}{4 \pi} & \equiv \int_{0}^{\pi} d \theta \sin ^{2} \theta \frac{U_{J}(\cos \theta)}{\cosh \omega+\cos \theta} \\
& =\frac{1}{2} \int_{-\pi}^{\pi} d \theta \frac{\sin \theta \sin (J+1) \theta}{\cosh \omega+\cos \theta} \\
& =\frac{1}{4} \int_{-\pi}^{\pi} d \theta \frac{\cos J \theta-\cos (J+2) \theta}{\cosh \omega+\cos \theta} \\
& =\frac{1}{4} \int_{-\pi}^{\pi} d \theta \frac{e^{i J \theta}-e^{i(J+2) \theta}}{\cosh \omega+\cos \theta} .
\end{aligned}
$$

Assume $\operatorname{Re} J>-1$ and write $z=e^{i \theta}$; the integral becomes a contour integral over a unit circle counterclockwise from $-1-i \epsilon$ to $-1+i \epsilon$. We take $|\arg z|<\pi$. The contour may be distorted into one running from $-1-i \epsilon$ around the origin and back to $-1+i \epsilon$, appropriately avoiding the singularity at $z=-e^{-\omega}$. With a little care, one finds

$$
\begin{aligned}
\frac{I(\cdot J)}{4 \pi}= & \pi \cos \pi J e^{-(J+1) \omega} \\
& +\sin \pi J \mathrm{P} \int_{0}^{1} d x \frac{x^{J}-x^{J+2}}{\left(e^{\omega}-x\right)\left(x-e^{-\omega}\right)},
\end{aligned}
$$

where $\mathbf{P}$ means the principal-value instruction at $x=e^{-\omega}$. Note that for integer $J$ the second term vanishes and we recover our old answer for $I_{J}(\omega)$.

Consider now the quantity $a(J)$ defined by

$$
a(J)=\frac{1}{2 \pi^{2}} \int d \Omega F(\cos \theta) \frac{\sin (J+1) \theta}{\sin \theta}
$$

which except for a factor of $(-1)^{J}$ is just $g_{J}$ if $J$ is integral [see Eq. (2.10)]. Here we want it for arbitrary $J$ with only the initial restriction $\operatorname{Re} J$ $>-1$. Evidently $a(J)$ is an entire function of $J$ since the integration extends over a finite region, and, barring pathological behavior of $F$, there can be no singularities in the finite $J$ plane. Next we substitute the dispersion representation (2.12) for $F$ and use the above result for $I(J)$ and find

$$
a(J)=\frac{\cos \pi J}{\pi P q} \int_{m^{2}}^{\infty} d \rho^{2} e^{-(J+1) \omega} F_{D}\left(\rho^{2}\right)+\frac{\sin \pi J}{2 \pi^{2} P q} \int_{0}^{1} d x x^{J-1}\left(1-x^{2}\right) \mathrm{P} \int_{m^{2}}^{\infty} d \rho^{2} \frac{F_{D}\left(p^{2}\right)}{\left(\rho^{2}+P^{2}+q^{2}\right) / 2 P q-\left(1+x^{2}\right) / 2 x}
$$

We write this as

$$
a(J)=\cos \pi J g_{J}+\sin \pi J \tilde{g}_{J},
$$

where $g_{J}$ is the extension of the partial-wave amplitude to complex $J$ that we introduced earlier [Eq. (2.15)] and $\tilde{g}_{J}$ may be written in terms of $F((P$ $\left.+q)^{2}\right)[\mathrm{Eq} .(2.12)]$ as

$$
\tilde{g}_{J}=\frac{1}{\pi} \int_{0}^{1} d x x^{J-1}\left(1-x^{2}\right) \tilde{F}\left(P^{2}+q^{2}-2 P q \frac{1+x^{2}}{2 x}\right),
$$

where

$$
\tilde{F}\left(\kappa^{2}\right)=F\left(\kappa^{2}+i \epsilon\right)+F\left(\kappa^{2}-i \epsilon\right) .
$$

This may be rewritten by a change of integration variable as

$$
\tilde{g}_{J}=\frac{1}{\pi P q} \int_{-(P-q)^{2}}^{\infty} d \rho^{2} e^{-(J+1) \omega} \tilde{F}\left(-\rho^{2}\right)
$$

where $P, q$ stand for the magnitudes of our Euclidean four-vectors and where again the formal relation between $\rho^{2}$ and $\omega$ used earlier obtains:

$$
\cosh \omega=\frac{\rho^{2}+P^{2}+q^{2}}{2 P q} \text {. }
$$

We now notice that the relation (2.17) can be extended by analytic continuation to the entire domain of analyticity of $g_{J}$ and $\tilde{g}_{J}$. From the original definition, (2.16), of $a(J)$ we observe the symmetry relation $a(J)+a(-J-2)=0$. Thus Eq. (2.17) implies

$$
\cos \pi J\left(g_{J}+g_{-J-2}\right)=-\sin \pi J\left(\tilde{g}_{J}-\tilde{g}_{-J-2}\right) .
$$

At integer values of $J, \sin \pi J$ vanishes. Thus one obtains the Lorentz symmetry relation

$$
\left.g_{J}+g_{-J-2}=0 \quad \text { (integer } J\right)
$$


provided that $\tilde{g}_{J}$ has no poles at integer values of $J$.

According to the representation (2.18), $\tilde{g}_{J}$ will have a pole on integer value of $J$, say $J=-N$, if the asymptotic expansion of $F\left((q+P)^{2}, q^{2}, P^{2}\right)$ for $(q+P)^{2} \rightarrow \infty$ contains a term ${ }^{10} a\left(q^{2}, P^{2}\right)\left[(q+P)^{2}\right]^{-N}$. We are imagining a world of anomalous dimensions, so we here assume that $F$ does not contain any terms that fall like integer powers of $(q+P)^{2}$. On this assumption the Lorentz symmetry condition (2.19) holds.

We close this section with the appropriate extension to complex $J$ of the remaining partialwave amplitudes $\chi_{J}^{i}(i=2,3,4)$ and their Lorentz symmetry conditions. In terms of the decomposition of $\chi$ in terms of $F_{1}, \ldots, F_{4}$ [Eq. (2.11)] we find, using our basis functions $G_{J}^{i}$ and recursion relations among the $U_{J}(\cos \theta)$, the following relations for integer $J$ (we repeat the definition of $\chi_{J}^{1}$ for completeness):

$$
\begin{aligned}
& \chi_{J}^{1}=P^{-J} g_{J}^{1}, \\
& \chi_{J}^{2}=P^{-J} q P\left(\frac{J}{J+2} g_{J+1}^{2}-g_{J-1}^{2}\right), \\
& \chi_{J}^{3}=\frac{1}{2} P^{-J}\left(-P g_{J-1}^{3}+\frac{J}{J+1} q g_{J}^{4}\right), \\
& \chi_{J}^{4}=\frac{1}{2} P^{-J}\left(-q g_{J}^{4}+\frac{J+1}{J+2} P g_{J+1}^{3}\right),
\end{aligned}
$$

where

$$
g_{J}^{i}=\frac{(-1)^{J}}{2 \pi^{2}} \int d \Omega F_{i} U_{J}(\cos \theta)
$$

Assuming a dispersion relation for each of the invariant amplitudes $F_{i}$, we can evidently write the appropriate extension of the $g_{J}^{i}$ into the complex $J$ plane in terms of the weight functions in the dispersion relations, just as we did in detail for $g_{J}^{1}$. Then Eq. (2.20) provides the definitions of $\chi_{J}^{i}$ for complex $J$. Assuming that no integer-power falloff occurs in the $F_{i}$, the functions $g_{J}^{i}$ will all obey the Lorentz symmetry equation

$$
g_{J}^{i}+g_{-J-2}^{i}=0
$$

for integer $J$. By combining Eqs. (2.20) and (2.22) one derives the following relations for the $\chi_{\delta}^{i}$ at integer $J$ :

$$
\begin{aligned}
& P^{J} \chi_{J}^{1}+P^{-J-2} \chi_{-J-2}^{1}=0, \\
& (J+2) P^{J} \chi_{J}^{2}-J P^{-J-2} \chi_{-J-2}^{2}=0, \\
& (J+1) P^{J} \chi_{J}^{3}-J P^{-J-2} \chi_{-J-2}^{4}=0 .
\end{aligned}
$$

These relations play an important role in our evaluation of the pion form factor $F\left(Q^{2}\right)$ : They ensure that the asymptotic expansion of $F\left(Q^{2}\right)$ for $Q^{2} \rightarrow \infty$ does not contain any terms that fall like integer powers of $Q^{2}$.

\section{THE LOOP INTEGRAL}

The form factor $F\left(Q^{2}\right)$ of the pion is defined in terms of the matrix element of the electromagnetic current by

$$
\left\langle P^{\prime}\left|J^{\mu}(0)\right| P\right\rangle=\left(P^{\prime \mu}+P^{\mu}\right) F\left(Q^{2}\right),
$$

where $Q^{\mu}=P^{\prime \mu}-P^{\mu}$. It contains a disconnected contribution [Fig. 2(a)] and a connected contribution [Fig. 2(b)], which contains all of the remaining graphs. Each contribution is separately gauge invariant, as will be shown below.

The analysis in this paper is confined to the disconnected graph. As we will see, the dominant contributions to the disconnected graph can be thought of as arising from two regions in the loop integration: (1) the short-distance region, in which $q^{2},(q+P)^{2}$, and $\left(q+P^{\prime}\right)^{2}$ are all large, and (2) the "wave-function pole" region in which only $(q+P)^{2}$ is large. We give a careful analysis of the contributions from these two regions in this paper. We also developed power-counting methods, of somewhat questionable validity, for calculating these contributions for the disconnected graph. These power-counting methods give results in agreement with the results of this paper for the disconnected graph. When we apply these same power-counting methods to the connected graph, Fig. 2(b), we find that the connected and disconnected graphs contribute the same leading powers of $Q^{2}$ to the asymptotic expansion of $F\left(Q^{2}\right)$. Thus we expect that careful examination of the disconnected graph alone is sufficient to determine the correct behavior of $F\left(Q^{2}\right)$. However, the careful determination of the behavior of the connected
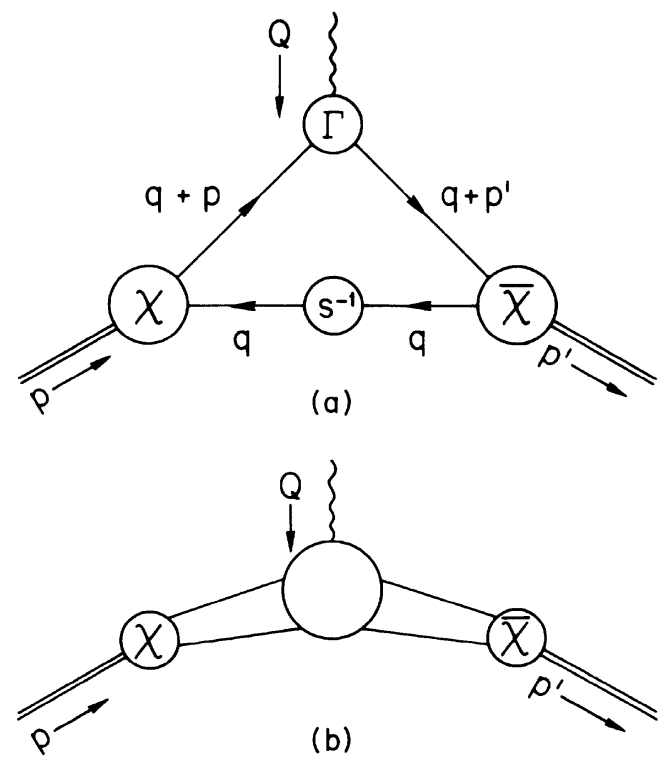

FIG. 2. Feynman diagrams for the pion form factor. 
graph remains an open problem.

In order to keep the initial discussion as simple as possible, we make two replacements in the disconnected graph. The full inverse quark propagator is replaced by a free inverse propagator $(i q+m)$ and the full electromagnetic vertex function of the quark is replaced by the bare vertex $\gamma^{\mu}$. The calculation using these approximations illustrates most of the essential physics of the problem and yields almost the correct answer. In the Appendix we will show how to extend the method of calculation to include the quark propagator and vertex corrections.

Thus, the amplitude to be investigated in this section is

$$
\left\langle P^{\prime}\left|J^{\mu}(0)\right| P\right\rangle \cong(2 \pi)^{-4} \int d^{4} q \operatorname{Tr}\left\{e \gamma^{\mu} \chi\left(q^{\nu}+P^{\nu}, q^{\nu}\right)[i q+m] \bar{\chi}\left(q^{\nu}, q^{\nu}+P^{\prime \nu}\right)\right\} .
$$

(There is, of course, another term in which the current acts on the antiquark line. This term can be treated in the same manner, and yields the same asymptotic behavior, as the term we consider.) For $\bar{\chi}$ one can substitute $-T^{-1} \chi^{T} T$ [see Eq. (2.5)].

One can easily show that this expression is gauge invariant by taking the transpose of the matrix in curly brackets, then using the properties of $T$ to restore the matrix to its original form except for the interchange of $P$ and $P^{\prime}$. Thus the expression must be proportional to the symmetric combination $P^{\prime \mu}+P^{\mu}$, which is also gauge invariant. The same argument may be used if the full propagator and vertex functions are included. (One uses $P T$ invariance to show that $T S^{-1}(q) T^{-1}=\left[S^{-1}(q)\right]^{T}$ and $\left.T \Gamma^{\mu}\left(P+q, P^{\prime}+q\right) T^{-1}=\Gamma^{\mu}\left(P^{\prime}+q, P+q\right)^{T}.\right)$

Multiplying Eq. (3.1) by $P_{\mu}$ and using the expression (3.2) for the current matrix element, one finds

$$
\begin{aligned}
F\left(Q^{2}\right)=- & \frac{2}{Q^{2}+4 M^{2}}(2 \pi)^{-4} \\
& \times \int d^{4} q \operatorname{Tr}\left\{\not p \chi\left(q^{\nu}+p^{\nu}, q^{\nu}\right)[i q q+m]\right. \\
& \left.\times \bar{\chi}\left(q^{\nu}, q^{\nu}+P^{\prime \nu}\right)\right\} .
\end{aligned}
$$

\section{Wick rotation}

We wish to evaluate $F\left(Q^{2}\right)$ as $Q^{2} \rightarrow \infty$. We begin by making a Wick rotation of ${ }^{11}$ the $d^{4} q$ integration:

$$
\int d^{4} q=\int d q^{0} d q^{1} d q^{2} d q^{3}-i \int d q^{4} d q^{1} d q^{2} d q^{3}
$$

where $q^{4}=i q^{0}$ is real after rotation of the integration contour counterclockwise through an angle of $\pi / 2$. In order to rotate the $q^{0}$ integration contour without meeting any cuts in the $q^{0}$ plane it is necessary to simultaneously analytically continue the amplitude to Euclidean values of the pion momenta $P^{\mu}$ and $P^{\prime \mu}$. We can do this with impunity because we have defined the wave functions $\chi$ off shell. After we have transformed the $q^{\nu}$ integration into a more tractable form, but before we take the $Q^{2} \rightarrow \infty$ limit, we will continue back to the pion mass shell, $P^{2}=P^{\prime 2}=-M^{2}$.

Thus, after Wick rotation we have

$$
F=-\frac{2 i}{Q^{2}+M^{2}}(2 \pi)^{-4} \int_{0}^{\infty} q^{3} d q \int d \Omega_{q} \operatorname{Tr}\left\{\not \boldsymbol{P} \chi\left(q^{\nu}+P^{\nu}, q^{\nu}\right)[i q+m] \bar{\chi}\left(q^{\nu}, q^{\nu}+P^{\prime \nu}\right)\right\},
$$

where $d \Omega_{q}$ is the surface area differential on a unit sphere in four dimensions.

\section{$\mathrm{O}(4)$ expansion}

We now substitute $-T^{-1} \chi^{T} T$ for $\bar{\chi}$ in the expression (3.4) for the form factor and insert the $O(4)$ expansion (2.7) for the two pion wave functions. Let $q \equiv\left(q^{2}\right)^{1 / 2}, \hat{q}^{\mu} / q$, with corresponding definitions for $P, P^{\prime}, \hat{P}^{\mu}$, and $\hat{P}^{\prime \mu}$. Then

$$
F=-\frac{2(2 \pi)^{-2}}{Q^{2}+4 M^{2}} \int_{0}^{\infty} q^{3} d q P q \sum_{J, L=0}^{\infty} \sum_{j, l=1}^{4} P^{J} \chi_{J}^{j}\left(q^{2}, P^{2}\right) C_{J L}^{j l}\left(m / q, \hat{P} \cdot \hat{P}^{\prime}\right) P^{\prime L} \chi_{L}^{l}\left(q^{2}, P^{\prime 2}\right),
$$

where

$$
C_{J L}^{j l}\left(m / q, \hat{P} \cdot \hat{P}^{\prime}\right)=\frac{-i}{(2 \pi)^{2}} \int d \Omega_{q} \operatorname{Tr}\left\{\hat{P} G_{J}^{j}\left(\hat{q}^{\nu}, \hat{P}^{\nu}\right)[i \hat{q}+m / q] T^{-1} G_{L}^{l}\left(\hat{q}^{\nu}, \hat{P}^{\prime \nu}\right)^{T} T\right\} .
$$


The angular integral and trace for $C_{J L}^{j l}$ can be calculated in closed form. One begins by noting that $G_{L}^{l}\left(\hat{q}^{\nu}, \hat{P}^{\prime \nu}\right)$ has the form

$$
G_{L}^{l}\left(\hat{q}^{\nu}, \hat{P}^{\prime v}\right)=\tilde{G}_{L}^{l}\left(\hat{q}^{\nu}\right)^{\mu_{1}} \cdots \mu_{L}\left\{\hat{P}_{\mu_{1}}^{\prime} \cdots \hat{P}_{\mu_{L}}^{\prime}\right\},
$$

where $\tilde{G}^{\mu_{1}} \cdots \mu_{L}$ is traceless and symmetric in $\mu_{1} \cdots \mu_{L}$. The factor $\left\{\hat{P}_{\mu_{1}}^{\prime} \cdots \hat{P}_{\mu_{L}}^{\prime}\right\}$ can be taken out of the integral, and the remaining integration must yield a traceless symmetric tensor constructed only from $\hat{P}^{\mu}$. Thus $C_{J L}^{j l}$ must have the form

$$
\left.C_{J L}^{j l}\left(m / q, \hat{P} \cdot \hat{P}^{\prime}\right)=\frac{2^{L}}{L+1} D_{J L}^{j l}(m / q)\left\{\hat{P}^{\mu_{1}} \cdots \hat{P}^{\mu_{L}}\right\} \hat{P_{\mu_{1}}^{\prime}} \cdots \hat{P}_{\mu_{L}}^{\prime}\right\}
$$

or

$$
C_{J L}^{j l}\left(m / q, \hat{P} \cdot \hat{P}^{\prime}\right)=\frac{1}{L+1} D_{J L}^{j l}(m / q) U_{L}\left(\hat{P} \cdot \hat{P}^{\prime}\right),
$$

where, as before,

$$
U_{L}(\cos \theta)=\frac{\sin [(L+1) \theta]}{\sin \theta}
$$

is a Chebyshev polynomial of the second kind. ${ }^{12}$ Now $D_{J_{L}}^{j l}(m / q)$ can be evaluated by considering the special case $\hat{P}^{\prime \nu}=\hat{P}^{\nu}$ :

$$
D_{J L}^{j l}(m / q)=C_{J L}^{j l}(m / q, 1) .
$$

One can easily show that

$$
D_{J L}^{j l}(m / q)=D_{L J}^{l j}(m / q)
$$

by taking the transpose of the matrix in curly brackets in Eq. (3.6) and using the properties of the matrix $T$. Furthermore, since $G_{J}^{j}$ and $G_{L}^{l}$ are basis functions for representations of $O(4)$ and $\hat{P}$ carries $O(4)$ angular momentum 1 , it is easy to see that $D_{J L}^{j l}$ vanishes unless $L=J \pm 1$. The nonvanishing integrals can be carried out explicitly ${ }^{13}$ and are given by

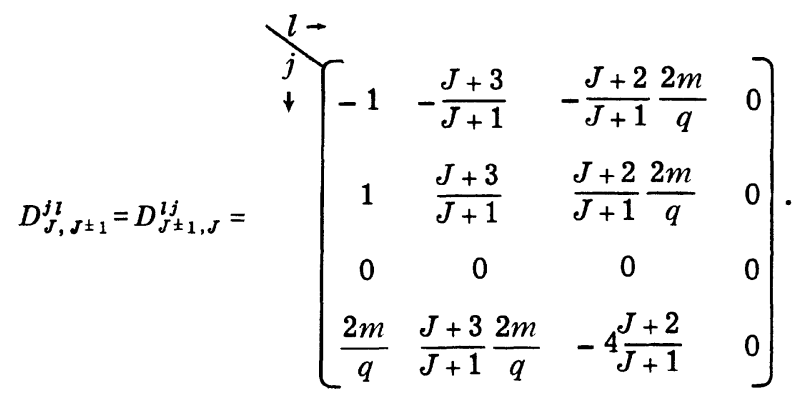

Thus, finally, setting $z \equiv P \cdot P^{\prime} / P P^{\prime}$,

$$
F=\frac{1}{Q^{2}+4 M^{2}}\left[-G\left(-1, P^{2}, P^{\prime 2}\right) U_{0}(z)-\sum_{J=0}^{\infty} G\left(J, P^{2}, P^{\prime 2}\right)\left(P P^{\prime}\right)^{J+1} U_{J+1}(z)\right],
$$

where the first term has been written separately for later convenience and

$$
G\left(J, P^{2}, P^{\prime 2}\right)=\frac{2(2 \pi)^{-2}}{J+2} \int_{0}^{\infty} d q q^{4} \sum_{j, l=1}^{4}\left\{\chi_{J}^{j}\left(q^{2}, P^{2}\right) D_{J, J+1}^{j l}(m / q) \chi_{J+1}^{l}\left(q^{2}, P^{\prime 2}\right)+P^{2} \chi_{J+2}^{j}\left(q^{2}, P^{2}\right) D_{J+2, J+1}^{j l}(m / q) \chi_{J+1}^{l}\left(q^{2}, P^{\prime 2}\right)\right\} .
$$

The first term in the braces in $G(J)$ occurs naturally only for $J \geq 0$. It can be included in $G(-1)$ because it vanishes at $J=-1$, as can be verified by using the explicit form (3.9) of $D_{J L}^{j l}$ and the "Lorentz symmetry" properties (2.23) of the wave functions $\chi$.
The sum over $J$ in Eq. (3.10) is convergent in the Euclidean region, where $z$ obeys $-1<z<1$. We wish to analytically continue this sum so that $P^{2}$ and $P^{\prime 2}$ are returned to their physical values, which gives $z=1+Q^{2} / 2 M^{2}>1$. The continuation in $z$ is accomplished by writing the sum over $J$ as a 
Sommerfeld-Watson integral in the complex $J$ plane. We will then investigate the limit $z \rightarrow \infty$. Thus, we will need to know how $U_{J}(z)$ behaves for complex $J$ and large $z$.

Analytic continuation of $U_{J}(z)$

Note that the function $U_{J}(\cos \theta)=\sin [(J+1) \theta] / \sin \theta$ can be written as

$$
U_{J}(z)=\frac{\left[z+\left(z^{2}-1\right)^{1 / 2}\right]^{J+1}-\left[z+\left(z^{2}-1\right)^{1 / 2}\right]^{-(J+1)}}{2\left(z^{2}-1\right)^{1 / 2}} .
$$

The factor $\left(z^{2}-1\right)^{1 / 2}$ can be defined with a cut drawn from $z=-1$ to $z=1$, and the factor $\left[z+\left(z^{2}\right.\right.$ $\left.-1)^{1 / 2}\right]^{J+1}$ can be defined with a cut drawn along the real axis from $-\infty$ to 1 . One then notes that the cut in $U_{J}(z)$ extends only from $-\infty$ to -1 , as the discontinuity vanishes for $-1<z<1$.

We are interested in the limit $z \rightarrow \infty$, for which the two terms in $U_{J}(z)$ have different asymptotic forms. It is useful to separate these terms, defining

$$
V_{J}(z) \equiv \frac{\left[z+\left(z^{2}-1\right)^{1 / 2}\right]^{J+1}}{2\left(z^{2}-1\right)^{1 / 2}}
$$

$$
\begin{aligned}
F=\frac{1}{Q^{2}+4 M^{2}}[ & -G\left(-1, P^{2}, P^{\prime 2}\right) V_{0}(z) \\
& \left.+\sum_{j=-1}^{\infty} G\left(J, P^{2}, P^{\prime 2}\right)\left(P P^{\prime}\right)^{J+1} V_{-J-3}(z)-\frac{i}{2} \int d J \frac{G\left(J, P^{2}, P^{\prime 2}\right)}{\sin \pi J} e^{i \pi J}\left(P P^{\prime}\right)^{J+1} V_{J+1}(z)\right],
\end{aligned}
$$

where initially the integration contour circles the positive real axis from $J=\infty-i \epsilon$ around $J=0$ and back to $J=\infty+i \epsilon$. The factor of $(-1)^{J}$ (from the residue of $\sin \pi J)$ has been inserted as $e^{i \pi J}$ so that the integrand is well behaved as $|\operatorname{Im} J| \rightarrow \infty$. Note that in this limit

$$
\left|\frac{e^{i \pi J} V_{J+1}(\cos \theta)}{\sin \pi J}\right| \propto \frac{e^{-(\pi-\theta) \operatorname{Im} J}}{e^{-\pi ! \operatorname{Im} J}}
$$

The function $G\left(J, P^{2}, P^{\prime 2}\right)$ is presumably analytic in the right half $J$ plane and falls off at least like a power for large $|J|$. Thus the contour can be opened up so that it runs just to the left of the with the cut defined as before, extending from $-\infty$ to 1 . Then

$$
U_{J}(z)=V_{J}(z)-V_{-J-2}(z) .
$$

As $|z| \rightarrow \infty$,

$$
V_{J}(z)-(2 z)^{J}
$$

\section{Sommerfeld-Watson transformation}

We are now prepared to analytically continue to the physical values of $P^{2}$ and $P^{\prime 2}$, and to extract the $Q^{2} \rightarrow \infty$ limit. Begin by substituting expression (3.14) for $U_{J}(z)$ into the expression (3.10) for $F$. [For $-1 \leq z \leq 1$, Eq. (3.14) is valid when the righthand side is evaluated for $z$ above or below the cut. For reasons that will be seen later, we choose to evaluate the function $V_{J}(z)$ below the cut.] The part of the expression for $F$ which is proportional to $V_{-J-3}$ will give us no trouble, and may be left as a sum. The part proportional to $V_{J+1}$ can be treated by converting the sum over $J$ into a contour integral in the complex $J$ plane by means of a Sommerfeld-Watson transformation:

$$
\begin{aligned}
F=\frac{1}{Q^{2}+4 M^{2}}[ & -G(-1) V_{0}\left(1+Q^{2} / 2 M^{2}\right) \\
& \left.-\sum_{J=-1}^{\infty}(-1)^{J}\left(M^{2}\right)^{J+1} G(J) V_{-J-3}\left(1+Q^{2} / 2 M^{2}\right)+\frac{i}{2} \int_{\operatorname{Re} J=-\epsilon} d J \frac{\left(M^{2}\right)^{J+1} G(J)}{\sin \pi J} V_{J+1}\left(1+Q^{2} / 2 M^{2}\right)\right]
\end{aligned}
$$

where $G(J) \equiv G\left(J,-M^{2},-M^{2}\right)$. Note that the integrand still falls off exponentially as $|\operatorname{Im} J| \rightarrow \infty$. It is straightforward to check that an exponential falloff was maintained at all stages during the analytic continuation. imaginary $J$ axis from $J=-\epsilon-i \infty$ to $J=-\epsilon+i \infty$. Until this point we were prevented from continuing the expression to physical values of $P^{\nu}$ and $P^{\prime \nu}$ by the fact that, for $z>1, V_{J+1}(z)$ grows like $(2 z)^{\operatorname{Re} J}$ as $\operatorname{Re} J \rightarrow \infty$. Now that the contour of integration has been distorted so that $\operatorname{Re} J$ is fixed, this problem no longer arises. To carry out the continuation, it is convenient to first set $P^{\prime 2}=P^{2}$. One then continues to $P^{2}=P^{\prime 2}=-M^{2}$ by reversing the Wick rotation, which means that the phase of $P^{2}$ decreases from 0 to $-\pi$. It follows that $\operatorname{Im} z<0$ during the continuation, which is why we have defined $V_{J+1}(z)$ by its value below the cut. The continuation is then straightforward, yielding
Displacement of the contour

The next step is to displace the integration contour of Eq. (3.17) some distance to the left (to, say, $\left.\operatorname{Re} J=-N-\frac{1}{2}\right)$. We will argue in the next sec- 
tion that $G(J)$ has poles in the left half $J$ plane. When the contour has been moved past such a "dynamical" pole at $J=J_{\alpha}$, a contribution

$$
F_{\alpha}\left(Q^{2}\right)=-\left[\underset{J=J_{\alpha}}{\operatorname{Res} G(J)}\right] \frac{\pi\left(M^{2}\right)^{J+1}}{\sin \pi J_{\alpha}} \frac{V_{J_{\alpha}+1}\left(1+Q^{2} / 2 M^{2}\right)}{Q^{2}+4 M^{2}}
$$

from the integral around the pole must be included in $F\left(Q^{2}\right)$. This contribution falls off like $\left(Q^{2}\right)^{J_{\alpha}}$ as $Q^{2} \rightarrow \infty$. Thus the rightmost poles in $G(J)$ generate the asymptotically dominant pieces of $F\left(Q^{2}\right)$.

There are also contributions to $F\left(Q^{2}\right)$ arising from the poles of $\sin \pi J$ at $J=-1,-2, \ldots$. These terms can be combined with the other terms of Eq. (3.17), which also involve integer values of $J$, to give a contribution

$$
F_{\mathrm{int}}\left(Q^{2}\right)=\frac{-M^{2}}{Q^{2}+4 M^{2}}\left[\sum_{J=0}^{N-2}(-1)^{J}\left(M^{2}\right)^{-J-2} G(-J-2) V_{-J-1}\left(1+Q^{2} / 2 M^{2}\right)+\sum_{J=1}^{\infty}(-1)^{J}\left(M^{2}\right)^{J-2} G(J-2) V_{-J-1}\left(1+Q^{2} / 2 M^{2}\right)\right] .
$$

However, the function $G(J)$ has a reflection property $\left(M^{2}\right)^{-J-2} G(-J-2)=-\left(M^{2}\right)^{J-2} G(J-2)$ for integer values $J=0,1,2, \ldots$. This property is a consesequence of the Lorentz reflection properties, Eq. (2.23), of the $\chi_{j}^{i}\left(q^{2}, P^{2}\right)$ that appear in $G(J){ }^{14}$ This leads to a cancellation of all the terms in $F_{\text {int }}\left(Q^{2}\right)$ except for the last sum for values of the index $J$ $\geq N-1$. (These surviving terms will of course disappear one by one if $N$ is increased.) Thus, the fixed integer-power terms all cancel out; the asymptotic form of $F\left(Q^{2}\right)$ is determined entirely by the "dynamical" poles, which in turn are determined by the behavior of the Bethe-Salpeter wave function.
We could close the section at this point, but the final answer can be expressed more neatly if we first apply the Sommerfeld-Watson transformation to the surviving terms in $F_{\text {int }}\left(Q^{2}\right)$ :

$$
\begin{aligned}
& F_{\text {int }}\left(Q^{2}\right) \\
& \quad=-\frac{i}{2} \int_{\operatorname{Re} J=N-3 / 2} d J \frac{\left(M^{2}\right)^{J-1} G(J-2)}{\sin \pi J} \frac{V_{-J-1}\left(1+Q^{2} / 2 M^{2}\right)}{Q^{2}+4 M^{2}} .
\end{aligned}
$$

By a redefinition of the variable of integration, this term can be combined with the other integral over the contour at $\operatorname{Re} J=-N-\frac{1}{2}$, to yield a single "background" integral:

$$
B\left(N ; Q^{2}\right)=\frac{M^{2}}{Q^{2}+4 M^{2}} \frac{i}{2} \int_{\operatorname{Re} J=-N-1 / 2} d J \frac{1}{\sin \pi J}\left[\left(M^{2}\right)^{J} G(J)+\left(M^{2}\right)^{-J-4} G(-J-4)\right] V_{J+1}\left(1+Q^{2} / 2 M^{2}\right) .
$$

As $Q^{2} \rightarrow \infty$ the background integral falls off faster than the pole terms $F_{\alpha}\left(Q^{2}\right)$. To see this note that $\left|V_{J}\left(1+Q^{2} / 2 M^{2}\right)\right|=V_{\mathrm{Re} J}\left(1+Q^{2} / 2 M^{2}\right)$, so

$$
\begin{aligned}
\left|B\left(N ; Q^{2}\right)\right| \leq \frac{1}{Q^{2}+4 M^{2}} V_{-N+1 / 2}\left(1+Q^{2} / 2 M^{2}\right) I & \\
& \sim\left(Q^{2}\right)^{-N-1 / 2} I \text { as } Q^{2} \rightarrow \infty,
\end{aligned}
$$

where

$$
\begin{aligned}
I=\frac{1}{2} \int_{\operatorname{Re} J=-N-1 / 2} \mid d J \frac{M^{2}}{\sin \pi J} & {\left[\left(M^{2}\right)^{J} G(J)\right.} \\
& \left.+\left(M^{2}\right)^{-J-4} G(-J-4)\right] \mid .
\end{aligned}
$$

In conclusion, we have found in this section that the form factor can be written as a sum of pole terms plus a background contribution:

$$
F\left(Q^{2}\right)=\sum_{-N-1 / 2<J_{\alpha}} F_{\alpha}\left(Q^{2}\right)+B\left(N, Q^{2}\right),
$$

where $F_{\alpha}\left(Q^{2}\right)$ [Eq. (3.18)] falls off like $\left(Q^{2}\right)^{J_{\alpha}}$ and $B\left(N, Q^{2}\right)$ [Eq. (3.19)] falls off at least as fast as $\left(Q^{2}\right)^{-N-1 / 2}$. The pole terms arise from the poles (at positions $\left.J=J_{\alpha}\right)$ of an integral $G\left(J,-M^{2},-M^{2}\right.$ ) [Eq. (3.11)] over the wave functions $\chi_{J}^{i}\left(q^{2},-M^{2}\right)$.
It now remains to investigate the poles in $J$ and the large $-q^{2}$ behavior of these wave functions.

\section{STRUCTURE OF THE WAVE FUNCTION}

In the previous section it was shown that the asymptotic behavior of the pion form factor is determined by the leading poles in the function $G(J)$, defined by the integral shown in Eq. (3.11). In this section we will discuss the properties of the wave function which must be known in order to find the location of these poles. The poles will arise from two different mechanisms.

The first mechanism is a divergence of the integral at large $q^{2}$. The location of these "short distance" poles is determined by the large $-q^{2}$ behavior of $\chi_{J}^{i}\left(q^{2}\right)$, which in turn is determined by the leading terms in the operator-product expansion for $\psi(0) \bar{\psi}(x)$. Thus, the location of the "short distance" poles will be expressed in terms of the anomalous dimensions of the operators which appear in the expansion.

The second mechanism is the presence of poles in the wave function $\chi_{J}^{i}\left(q^{2}\right)$. As discussed in Sec. II [following Eq. (2.15)], these poles are deter- 
mined by the behavior of $\chi\left(q^{\nu}+P^{\nu}, q^{\nu}\right)$ as $(q+P)^{2}$ $\rightarrow \infty$ with $q^{2}$ fixed. Thus, in terms of the original loop integral in Minkowski space, these terms correspond to the region of integration in which only one leg is far off the mass shell. Unfortunately, we are unable to reliably determine the behavior of the wave function in this limit. If $q^{2}$ were also asymptotic, then the form of the wave function would be governed by conformal invariance, and the necessary limit could be extracted. In order to obtain an answer, we will have to assume that even for modest $q^{2}$ the behavior of the wave function as $(q+P)^{2} \rightarrow \infty$ is the same as the behavior of the conformally invariant wave function. More precisely, the conformally invariant wave function is a linear sum of computable functions $\chi_{\alpha \beta}^{\text {conformal, } s}\left(q^{\nu}+P^{\nu}, q^{\nu}\right)$, corresponding to the different operators $₫$ which appear in the operatorproduct expansion. We assume that as $(q+P)^{2} \rightarrow \infty$ with $q^{2}$ fixed

$\chi_{\alpha \beta}\left(q^{\nu}+P^{\nu}, q^{\nu}\right) \rightarrow \sum_{\delta} \chi_{\alpha \gamma}^{\text {conformal, } s}\left(q^{\nu}+P^{\nu}, q^{\nu}\right) f_{\gamma \beta}\left(\mathcal{S}, q^{\nu}\right)$.

[We allow for a matrix factor on the right because we know that $\chi_{\alpha \beta}$ has a physical (i.e., massive) propagator on the leg of momentum $q$, while $\chi_{\alpha \beta}^{\text {conformal }}$ has a massless propagator. Of course it is likely that $f_{\gamma \beta}\left(\delta, q^{\nu}\right)$ does more than simply correct for the propagator.] We emphasize that we know of no model-independent proof of the regularity property (4.1). Thus, we, along with Callan and Gross ${ }^{3}$ and Menotti, ${ }^{4}$ must adopt Eq. (4.1) as an assumption in order to proceed.

In view of the evident ad hoc nature of regularity assumption (4.1), we have investigated its validity using the ladder-model Bethe-Salpeter equation with scalar-gluon exchange. In this model we are able to compute the positions and residues of the rightmost poles of $\chi_{J}^{i}\left(q^{2}\right)$. We find that the wave function does satisfy Eq. (4.1). We will report on this investigation in a separate publication. ${ }^{5}$

It is worthwhile to note that if further analysis should indicate that $\chi_{J}^{i}\left(q^{2}\right)$ is not quite as nicely behaved as indicated by the ladder model (so that, for instance, poles become cuts), it would be an easy matter to insert the improved information about the wave function into Eq. (3.17) to find how $F\left(Q^{2}\right)$ is modified.

\section{Operator-product expansion}

We now begin the analysis of $\chi_{J}^{i}\left(q^{2}\right)$ for large $q^{2}$ by writing down the operator-product expansion ${ }^{15}$ for two Dirac fields:

$$
\begin{aligned}
& T\{\psi(0) \bar{\psi}(x)\}=\sum_{\delta} \sum_{J=J_{0}(\delta)}^{\infty} x^{p(s)} C_{J}^{1}\left(s ; x^{2}\right) \gamma_{5}\left\{x^{\mu_{1}} \cdots x^{\mu}\right\} s_{\mu_{1}}^{(J)} \cdots \mu_{J} \\
& +\sum_{\delta} \sum_{J=\max }^{\infty} x_{\left.J_{0}(\delta), 1\right]}^{p(\delta)} C_{J}^{2}\left(\delta ; x^{2}\right)\left[\gamma^{\alpha}, \gamma^{\beta}\right] \gamma_{5}\left\{x^{\mu_{1}} \cdots x^{\mu_{J-1}} x_{\alpha}\right\} s_{\mu_{1} \cdots \mu_{J-1} \beta}^{(J)} \\
& +\sum_{\delta} \sum_{J=\max \left\{J_{0}(\delta), 1\right]}^{\infty} x^{p(\delta)+1} C_{J}^{3}\left(\delta, x^{2}\right) \gamma^{\lambda} \gamma_{5}\left\{x^{\mu_{1}} \cdots x^{\mu}{ }_{J-1}\right\} S_{\mu_{1} \cdots \mu_{J-1} \lambda}^{(J)} \\
& +\sum_{\delta} \sum_{J=J_{0}}^{\infty}(\delta) x^{p(\delta)-1} C_{J}^{4}\left(\delta, x^{2}\right) \gamma^{\lambda} \gamma_{5}\left\{x^{\mu_{1}} \cdots x^{\mu} J x_{\lambda}\right\} \mathcal{S}_{\mu_{1}}^{(J)} \cdots_{\mu_{J}} \\
& +\cdots \cdot
\end{aligned}
$$

Here $S$ is a local operator with $J_{0}(\delta)$ tensor indices, $S(x)_{\mu_{1} \cdots \mu_{J_{0}}}$, that is symmetric and traceless in those indices. For instance, one possible $\delta$ is $\bar{\psi}(\chi) \gamma_{5}\left\{\ddot{\partial}_{\mu_{1}} \cdots \vec{\partial}_{\mu_{J_{0}}}\right\} \psi(x)$. The operators $\boldsymbol{S}^{(J)}$ are formed from $S$ by differentiating $J-J_{0}$ times and taking the traceless symmetric part:

$$
\mathcal{S}_{\mu_{1} \cdots \mu_{J}}^{(J)}=\left\{\partial_{\mu_{J_{0}+1}} \cdots \partial_{\mu_{J}} \delta(0)_{\mu_{1} \cdots \mu_{J_{0}}}\right\} .
$$

Since we are expanding the time-ordered product of the two fields, $x^{p}$ is to be interpreted as $\left(x^{\mu} x_{\mu}\right.$ $+i \epsilon)^{p / 2}$. The exponent $p(\delta)$ is to be chosen by dimension counting so that the remaining functions $C_{J}^{i}\left(\delta ; x^{2}\right)$ approach constants $C_{J}^{i}(\delta)$ as $x^{2} \rightarrow 0$ :

$$
p(\S)=d_{\S}-J_{0}(\S)-2 d_{\psi},
$$

where $d_{S}$ and $d_{\downarrow}$ are the dimensions of $\delta_{\mu_{1}} \cdots_{J_{0}}$ and $\psi$, respectively.

There is a sum over possible operators $S$. The dots indicate other terms in the operator-product expansion that do not contribute to the Bethe-Salpeter wave function $\langle 0|T\{\psi(0) \bar{\psi}(x)\}| P\rangle$. (The noncontributing terms involve operators that either have the wrong parity or are not fully symmetric in their indices.)

\section{Implications of conformal invariance}

If we take $x^{2}=0$ in the coefficients $C_{J}^{i}\left(S, x^{2}\right)$ we obtain ${ }^{16,17,3}$ the coefficients $C_{J}^{i}(S)$ of the massless theory (in our case, the massless $g \bar{\psi} \psi \phi$ theory) 
evaluated at the fixed point of the renormalization group. ${ }^{18}$ Since this massless theory is conformally invariant, there must be relations among the coefficients $C_{J}^{i}(S)$ that reflect conformal invariance. These relations can be derived by applying the methods developed by Ferrara, Grillo, and Gatto $^{19}$ for the operator-product expansion of two scalar fields.

We commute the conformal generator $K_{\nu}$ with both sides of the operator-product expansion. On the left-hand side we use

$$
\begin{aligned}
& {\left[T\{\psi(0) \bar{\psi}(x)\}, K_{\nu}\right]} \\
& \quad=T\{\psi(0) \bar{\psi}(x)\}\left(2 i d_{\psi} x_{\nu}-\vec{i} \overleftarrow{\partial}_{\nu} x^{2}+2 \vec{i} \overleftarrow{\partial} \cdot x x_{\nu}-\frac{1}{2}\left[\gamma_{\nu}, \not x\right]\right) .
\end{aligned}
$$

We insert the operator-product expansion for $T\{\psi(0) \bar{\psi}(x)\}$ and apply the differential operator indicated to the $x$ 's and $\gamma$ matrices in each term. On the right-hand side we use

$$
\left[\delta_{\mu_{1}}^{(J)} \cdots \mu_{J}, K^{\nu}\right]=2 i\left(J-J_{0}\right)\left(J+d_{S}-1\right)\left\{\delta_{\mu_{1}}^{(J-1)} \mu_{\mu_{J}-1} g_{\mu_{J}}^{\nu}\right\},
$$

where the traceless-symmetric operation on the right-hand side applies to the indices $\mu$, but not to $\nu$. We then collect the results into terms that transform under the various irreducible representations of the Lorentz group, ignore those representations that do not contribute to matrix elements between the one-pion state and the vacuum, and in each remaining term ignore pieces that are of higher order in $x^{2}$. This gives us four recursion relations for the four sets of coefficients $C_{J}^{1}(S)$, $C_{J}^{2}(\S), C_{J}^{3}(\S)$, and $C_{J}^{4}(\S)$ that correspond to each independent operator. (The details of this calculation, including a treatment of mixed symmetry operators that do not contribute to the pion wave function, will be given in a separate publication. ${ }^{20}$ ) The four recursion equations are

$$
\left[\begin{array}{cc}
2 J+d_{\delta}-J_{0} & 2 \\
\frac{1}{2} & 2 J+d_{S}-J_{0}
\end{array}\right]\left(\begin{array}{l}
C_{J}^{1}(\S) \\
C_{J}^{2}(\S)
\end{array}\right)=2\left(J+1-J_{0}\right)\left(J+d_{S}\right)\left(\begin{array}{l}
C_{J^{+1}}^{1}(\S) \\
C_{J+1}^{2}(\S)
\end{array}\right)
$$

and

$$
\left[\begin{array}{cc}
2 J+d_{\S}-J_{0} & \frac{2 J+d_{\S}-J_{0}}{2(J+1)(J+2)} \\
0 & 2 J+d_{\S}-J_{0}
\end{array}\right]\left(\begin{array}{l}
C_{J}^{3}(\S) \\
C_{J}^{4}(\S)
\end{array}\right)=2\left(J+1-J_{0}\right)\left(J+d_{\S}\right)\left(\begin{array}{l}
C_{J+1}^{3}(\S) \\
C_{J+1}^{4}(\S)
\end{array}\right) .
$$

We note immediately that the four recursion equations in four unknowns break up into two sets of two equations, one for $C_{J}^{1}, C_{J}^{2}$ and one for $C_{J}^{3}, C_{J}^{4}$. This is consistent with the requirement of chiral invariance that $C_{J}^{1}(\delta)=C_{J}^{2}(\delta)=0$ for evenchirality operators $S$ and $C_{J}^{3}(S)=C_{J}^{4}(\$)=0$ for oddchirality operators $S$. (One proves this rule by noting that the zero-mass $\bar{\psi} \psi \phi$ theory is invariant under the chiral transfor mation $U$ with $U^{-1} \psi U=\gamma_{5} \psi$, $U^{-1} \bar{\psi} U=-\bar{\psi} \gamma_{5}, U^{-1} \phi U=-\phi$. Thus

$$
U^{-1} T\{\psi(0) \bar{\psi}(x)\} U=-\gamma_{5} T\{\psi(0) \bar{\psi}(x)\} \gamma_{5} .
$$

When we insert the operator-product expansion for $T\{\psi(0) \bar{\psi}(x)\}$ we learn that the sign of the $\gamma$-matrix part of each term under $\Gamma \rightarrow-\gamma_{5} \Gamma \gamma_{5}$ must be the same as the sign of the operator $\delta^{(J)}$ under $\delta^{(J)}$ $\rightarrow U^{-1} g^{(J)} U$, or else the coefficient $C_{J}^{i}(\delta)$ of that term must vanish.)

The recursion equations are easily solved, but we can see where the poles in $J$ of the solutions are even without solving the equations. We assume that $C_{J}^{i}(\delta)$ is analytic in the right half $J$ plane, and use the recursion equations to continue $C_{J}^{i}$ into the left-hand plane in steps of $\Delta J=-1$. Poles in $C_{J}^{i}$ occur at those points $J_{\delta}$ where the matrix in the recursion equation becomes singular (and at $J_{S}-1$, $J_{s}-2$, etc.).

In the case of an odd-chirality operator $\delta$, the nonzero coefficients are $C_{J}^{1}$ and $C_{J}^{2}$. They become singular when

$$
\left(2 J+d_{\S}-J_{0}\right)^{2}-1=0
$$

that is, when $J=J_{\mathcal{E}}$, where

$$
J_{\delta}=+\frac{1}{2}-\frac{1}{2}\left[d_{S}-J_{0}(S)\right]
$$

or when $J=-\frac{1}{2}-\frac{1}{2}\left[d_{S}-J_{0}(\delta)\right]$, which is $J=J_{\delta}-1$. (There are double poles at $J_{S}-1, J_{\delta}-2, \ldots$; these lead to $\log Q^{2}$ factors in the expansion of the form factor.)

In a $g \bar{\psi} \psi \phi$ theory the lowest-twist odd-chirality operators with nonvanishing matrix elements between the one-pion state and the vacuum are

$$
\bar{\psi}(x) \gamma_{5}\left\{\vec{\partial}_{\mu_{1}} \cdots \bar{\partial}_{\mu_{J_{0}}}\right\} \psi(x), J_{0}=0,2,4, \ldots
$$

and

$$
\bar{\psi}(x) \gamma_{5}\left\{\gamma_{\mu_{1}} \vec{\partial}_{\mu_{2}} \cdots \vec{\partial}_{\mu_{J_{0}}}\right\} \psi(x) \phi(x), \quad J_{0}=1,3, \ldots
$$

These operators have twist 3 , so (for smali anom- 
alous dimensions) $d_{S}-J_{0} \simeq 3$ and $J_{S}=+\frac{1}{2}-\frac{1}{2}\left(d_{S}-J_{0}\right)$ $\simeq-1$. In the $g \bar{\psi} \psi \phi$ ladder model we $\operatorname{find}^{21} d_{\psi}=\frac{3}{2}$ and

$$
\begin{aligned}
d_{S} & =2+\left[\left(J_{0}+1\right)^{2}-g^{2} /(2 \pi)^{2}\right]^{1 / 2} \\
& \simeq 3+J_{0}-\frac{1}{J_{0}+1} \frac{g^{2}}{8 \pi^{2}}+O\left(g^{4}\right)
\end{aligned}
$$

for $S=\bar{\psi} \gamma_{5}\left\{\partial_{\mu} \cdots \partial_{\mu}\right\} \psi$ and

$$
\begin{aligned}
d_{S} & =2+\left[\left(J_{0}+1\right)^{2}+g^{2} /(2 \pi)^{2}\right]^{1 / 2} \\
& =3+J_{0}+\frac{1}{J_{0}+1} \frac{g^{2}}{8 \pi^{2}}+O\left(g^{4}\right)
\end{aligned}
$$

for $\bar{\psi} \gamma_{5}\left\{\gamma_{\mu} \partial_{\mu} \cdots \partial_{1}\right\} \psi \phi$.

The residue of $C_{J}^{i}(\delta)$ at the pole $J=J_{S}$ is also easily computed (up to a normalization constant) directly from the recursion equation. At $J=J_{S}$ the matrix multiplying $C_{J}^{i}$ is

$$
\left(\begin{array}{ll}
1 & 2 \\
\frac{1}{2} & 1
\end{array}\right)
$$

This matrix annihilates the vector $C^{i}=(2,-1)$. Thus

$$
\underset{J=J S}{\operatorname{Res}} C_{J}^{i}(\delta)=\text { const } \times(2,-1,0,0) .
$$

Let us turn to the case of an even-chirality operator $\delta$, with corresponding nonzero coefficients $C_{J}^{3}$ and $C_{J}^{4}$. According to the recursion equation, these coefficients have a pole at $J=J_{S}$ where

$$
J_{\delta}=-\frac{1}{2}\left[d_{\delta}-J_{0}(\delta)\right] .
$$

(Further poles then occur at $J=J_{S}-1, J_{\delta}-2$, etc.).

In a $g \bar{\psi} \psi \phi$ theory the lowest-twist even-chirality operators that contribute to the pion wave function are

$$
\bar{\psi}(x) \gamma_{5}\left\{\gamma_{\mu_{1}} \bar{\partial}_{\mu_{2}} \cdots \bar{\partial}_{\mu_{J_{0}}}\right\} \psi(x), \quad J_{0}=1,3,5, \ldots
$$

These operators have twist 2 , so, for small anomalous dimensions, $d_{\delta}-J_{0}(\delta) \simeq 2$ and $J_{\mathcal{S}} \simeq-1$. In the $g \bar{\psi} \psi \phi$ ladder model we find $^{21}$

$$
\begin{aligned}
d_{S}= & 2+\left\{J_{0}^{2}+2 J_{0}+2\right. \\
& \left.-2\left[\left(J_{0}+1\right)^{2}-\frac{g^{2}}{8 \pi^{2}}\left(2-\frac{g^{2}}{8 \pi^{2}}\right)\right]^{1 / 2}\right\}^{1 / 2} \\
= & 2+J_{0}+\frac{1}{J_{0}\left(J_{0}+1\right)} \frac{g^{2}}{8 \pi^{2}}+O\left(g^{4}\right) .
\end{aligned}
$$

The residue at $J=J_{S}$ of $C_{J}^{i}(\delta)$ is not determined by the conformal recursion equation in the case of an even-chirality operator $\delta$. There are two linearly independent solutions of the recursion equation, as in the odd-chirality case. However, the matrix multiplying $C_{J}^{i}(\delta)$ in the even-chirality recursion equation becomes identically zero at $J=J_{S}$ instead of annihilating just one vector $C_{J}^{i}$. Thus, the two independent solutions have different residues. What linear combination of the two conformally invariant solutions $C_{J}^{i}$ actually occurs must be determined by the Bethe-Salpeter equation. Using the laddermodel BS equation we find, ${ }^{21}$ at $J=J_{S}$,

$$
\operatorname{Res}_{J=J_{S}} C_{J}^{i}(S)=\text { const } \times\left(0,0, g^{2} / 8 \pi^{2}, 1-\left(d_{S}-J_{0}-3\right)^{2}\right) \text {. }
$$

We can now translate our information about the operator-product expansion (4.2) into information about the pion bound-state wave function. We take the matrix element of the operator-product expansion between the vacuum and one-pion states and the Fourier transform. The matrix elements of the operators $S_{\mu_{1}}^{(J)} \cdots \mu_{J}$ have the form

$$
\left.\left\langle 0\left|\boldsymbol{s}_{\mu_{1}}^{(J)} \cdots \mu_{J}\right| P\right\rangle=i^{J}\left\{P_{\mu_{1}} \cdots P_{\mu_{J}}\right\} \varsigma s\right\rangle,
$$

where $\langle s\rangle$ is a constant that depends cnly on the base operator $\delta_{\mu_{1}} \cdots \mu_{J}$ of the series to which $S_{\mu_{1}}^{(J)} \cdots \mu_{J}$ belongs. The ${ }^{J}$ Fourier transforms of the factors $x^{p}\left\{x^{\left.\mu_{1} \cdots x^{\mu_{L}}\right\}}\right.$ are certain constants times $q^{-4-L-p}\left\{q^{\mu_{1}} \cdots q^{\mu_{L}}\right\}$. Comparing the result to the definition Eq. (2.7) of the partial-wave amplitudes $\chi_{J}^{i}\left(q^{2}\right)$, we can read off the $q^{2} \rightarrow \infty$ asymptotic expansion of $\chi_{J}^{i}\left(q^{2}\right)$ corresponding to the $x^{2} \rightarrow 0$ operator-product expansion:

$$
\chi_{J}^{i}\left(q^{2}\right) \sim \sum_{\delta} a_{J}^{i}(\delta) C_{J}^{i}(\delta) q^{-4-J-p(\delta)}+\cdots,
$$

where the dots indicate terms of higher order in $q^{2}, p(s)=d_{s}-J_{0}-2 d_{\psi}$, and

$$
\begin{aligned}
& a_{J}^{1}(s)=16 \pi^{2} 2^{p(s)}\langle s\rangle \frac{\Gamma\left(J+2+\frac{1}{2} p(s)\right)}{\Gamma\left(-\frac{1}{2} p(s)\right)}, \\
& a_{J}^{2}(s)=-2 a_{J}^{1}(s), \\
& a_{J}^{3}(s)=16 \pi^{2} 2^{p(s)}\langle s\rangle \frac{\Gamma\left(J+\frac{3}{2}+\frac{1}{2} p(s)\right)}{\Gamma\left(-\frac{1}{2} p(s)-\frac{1}{2}\right)}, \\
& a_{J}^{4}(s)=8 \pi^{2} 2^{p(s)}\langle s\rangle \frac{\Gamma\left(J+\frac{5}{2}+\frac{1}{2} p(s)\right)}{\Gamma\left(-\frac{1}{2} p(\delta)+\frac{1}{2}\right)} .
\end{aligned}
$$

We have found that the coefficients $C_{J}^{i}(\S)$ in the operator-product expansion have poles in the left half $J$ plane. The coefficients $a_{J}^{i}(\delta)$ that arise in the Fourier transform to momentum space also have some poles in the left half $J$ plane. ${ }^{22}$ Let us denote the positions of the poles of the product $a_{J}^{i}(\delta) C_{J}^{i}(\delta)$ by $J(S, n)(n=1,2, \ldots$,$) , and the corre-$ sponding residues by $r^{i}(\delta, n)$. Thus the leading terms in $\chi_{J}^{i}\left(q^{2}\right)$ as $q^{2} \rightarrow \infty$ have the form

$$
\sum_{\delta, n} \frac{r^{i}(\delta, n)}{J-J(\delta, n)} q^{-4-J(\delta, n)-p(\delta)} \text {. }
$$


This $J$-plane structure has been determined by applying conformal invariance at large values of $q^{2}$. As explained at the beginning of this section, we assume that this structure is modified only by multiplication on the right by a matrix function $f_{\gamma \beta}\left(\delta, q^{\nu}\right)$, as shown in Eq. (4.1). Let this function be written as

$$
f\left(S, q^{\nu}\right)=f^{(1)}\left(\delta, q^{\nu}\right)+f^{(2)}\left(\delta, q^{\nu}\right) i q .
$$

Then note that multiplication of a wave function by $i q$ on the right has the following effect on the par- tial waves: If $\tilde{\chi}\left(q^{\nu}+P^{\nu}, q^{\nu}\right)=\chi\left(q^{\nu}+P^{\nu}, q^{\nu}\right) i q$, then

$$
\tilde{\chi}_{J}^{i}\left(q^{2}\right)=q \sum_{J} \chi_{J}^{j}\left(q^{2}\right) M_{J}^{j i}
$$

where the matrix $M_{J}^{i j}$ is defined by

$$
G_{J}^{i}\left(q^{\nu}, P^{\nu}\right) i q q=q \sum_{j} M_{J}^{i j} G_{J}^{j}\left(q^{\nu}, P^{\nu}\right)
$$

The matrix can be worked out explicitly using the identities of Appendix B of Ref. 7. and is given by

$$
M_{J}^{i j}=\frac{1}{2(J+1)}\left[\begin{array}{cccc}
0 & 0 & J & -(J+1) \\
0 & 0 & (J+2) & (J+1) \\
-2(J+1) & -2(J+1) & 0 & 0 \\
2(J+2) & -2 J & 0 & 0
\end{array}\right] .
$$

The assumption of Eq. (4.1) can now be rewritten as a direct statement about the leading poles of $\chi_{J}^{i}\left(q^{2}\right)$ :

$$
\begin{aligned}
\chi_{J}^{i}\left(q^{2}\right)= & \sum_{\boldsymbol{s}, n, j} \frac{r^{j}(\delta, n) q^{-4-J(s, n)-p(\$)}}{J-J(\delta, n)} \\
& \quad \times\left[f^{(1)}\left(\delta, q^{2}\right) \delta^{j i}+q f^{(2)}\left(\delta, q^{2}\right) M_{J}^{j i}\right] \\
& +\tilde{\chi}_{J}^{i}\left(q^{2}\right)
\end{aligned}
$$

where $\tilde{\chi}_{J}^{i}\left(q^{2}\right)$ is analytic in the region of interest, and as $q^{2} \rightarrow \infty, f^{(1)}-1$ and $q f^{(2)} \rightarrow 0$. We are assuming that Eq. (4.15) accurately describes at least the leading poles (i.e., those which occur near $J=-1)$.
It is apparent that the structure (4.15) is consistent with the large $-q^{2}$ form (4.13). A number of possible modifications of Eq. (4.15) that are equally consistent with Eq. (4.13) spring to mind. One might imagine that the pole positions are functions $J\left(q^{2} ; \varsigma, n\right)$ of $q^{2}$ with $J\left(q^{2} ; \varsigma, n\right) \rightarrow J(\varsigma, n)$ as $q^{2} \rightarrow \infty$. One might also imagine that the matrices $f^{(1)} \delta^{j i}$ $+q f^{(2)} M_{J}^{j i}$ that multiply the residues are more general matrix functions $A^{j i}\left(\mathcal{S}, q^{2}\right)$ that tend to $\delta^{j i}$ as $q^{2} \rightarrow \infty$. There are also more exotic possibilities. One could easily discuss the implications for $F\left(Q^{2}\right)$ of any of these possibilities. However, we will restrict our discussion to the simplest case represented by Eq. (4.15), as suggested by the ladder model.

\section{EVALUATION OF THE FORM FACTOR}

We must now use our information about $\chi_{J}^{i}\left(q^{2}\right)$ to find the poles in the integral $G(J)$ defined in Eq. (3.11):

$$
G(J)=\frac{2(2 \pi)^{-2}}{J+2} \int_{0}^{\infty} d q q^{4} \sum_{i, j=1}^{4}\left\{\chi_{J}^{j}\left(q^{2}\right) D_{J, J+1}^{j l} \chi_{J+1}^{l}\left(q^{2}\right)-M^{2} \chi_{J+2}^{j}\left(q^{2}\right) D_{J, J+1}^{j l} \chi_{J+1}^{l}\left(q^{2}\right)\right\},
$$

where the matrix $D_{J, J+1}^{j l}\left(q^{2} / m^{2}\right)$ is given in Eq. (3.9). [Recall that a pole in $G(J)$ at $J=J_{\alpha}$ produces a term $F_{\alpha}\left(Q^{2}\right)$ in the form factor that falls off like $\left(Q^{2}\right)^{J} \alpha$ as $Q^{2} \rightarrow \infty$.] We will be concerned only with the leading group of poles in $G(J)$, which occur near $J=-1$.

Poles in $G(J)$ can arise directly from poles in $\chi_{J}^{j}\left(q^{2}\right)$ or they can arise from a divergence of the integral at large $q^{2}$.

Consider first the poles in $G(J)$ produced directly from poles in $\chi_{J}^{j}\left(q^{2}\right)$ at $J=J(S, n)$, beginning with those poles associated with an odd-chirality operator $S$. The leading such poles in $\chi_{J}^{j}\left(q^{2}\right)$ occur at

$$
J=-1-\frac{1}{2} \gamma_{s},
$$

where $\gamma_{S}$ is the anomalous dimension of $S$ [see Eq. (4.7)]. Thus these poles in $\chi$ produce poles in $G(J)$ near $J=-1$; they also produce poles in $G(J)$ near $J=-2$ and $J=-3$, but we do not consider these nonleading poles.

The residue of $\chi_{J}^{i}\left(q^{2}\right)$ at one of these poles is given by Eq. (4.15) with 


$$
r^{j}=\text { const } \times(1,1,0,0),
$$

which we obtain by combining the conformal-invariance result (4.8) with the kinematical factors described by Eqs. (4.11) and (4.12). However, when we refer to $M^{j k}$ [Eq. (4.14)] and $D^{k l}$ [Eq. (3.9)] we notice that ${ }^{23}$

$$
\begin{aligned}
\sum_{j}\left[\operatorname{Res}_{J=J \mathbf{S}} \chi_{J}^{j}\left(q^{2}\right)\right] D_{J, J+1}^{j l}(q / m) \\
\quad=q^{-4-J} \mathcal{S}-p(\mathcal{S}) \sum_{j, k} r^{j}\left[f^{(1)} \delta^{j k}+q f^{(2)} M_{J}^{j k}\right] D_{J, J+1}^{k l} \\
\quad=0 .
\end{aligned}
$$

Thus $G(J)$ does not have poles at the locations $J=J_{S}$ of poles in the wave function associated with oddchirality operators $\$$.

This cancellation was first noticed by Menotti, ${ }^{4}$ who used a different method of calculation. (He actually discusses only the contribution from $S=\bar{\psi} \gamma_{5} \psi$, but his method can easily be generalized.) The cancellation can easily be seen in momentum space by noting that the odd-chirality part of the conformal-invariant wave function ${ }^{3,4}$ has the asymptotic form

$$
\chi\left(q^{\nu}+P^{\nu}, q^{\nu}\right) \sim\{2 q \cdot P+[\not P, \not q]\}
$$

as $(q+P)^{2} \rightarrow \infty$ with $q^{2}$ fixed. When multiplied on the left by $P$ [as it appears in Eq. (3.3) for the form factor], one can see immediately that the dominant term is canceled exactly. This cancellation is a property of the vector vertex function, and will not occur for a scalar form factor. It should also be noted that this cancellation depends crucially on the precise Dirac matrix structure of the wave function in the appropriate limit. If this structure were to be modified more than is allowed by Eq. (4.15), then then the cancellation would disappear, although the other conclusions of this paper would remain unchanged.

Consider now the poles in $G(J)$ produced by poles in $\chi_{J}^{i}\left(q^{2}\right)$ associated with even-chirality operators. The leading such poles are associated with the operators $\boldsymbol{S}=\bar{\psi} \gamma_{5}\left\{\gamma_{\mu} \partial_{\mu} \cdots \partial_{\mu}\right\} \psi$ and occur at

$$
J=-1-\frac{1}{2} \gamma_{\mathrm{s}},
$$

where again $\gamma_{\delta}$ is the anomalous dimension of $\delta$. Conformal invariance does not determine the residue of $\chi_{J}^{i}$ at a pole associated with an even-chirality operator, so we cannot guarantee that no cancellation occurs in the product $\chi_{J}^{j} D^{j l}$. However, no such cancellation occurs in the ladder model. Thus we may presume that $G(J)$ does indeed have poles at the locations $J=-1-\frac{1}{2} \gamma_{s}$.

There are also poles in $\chi_{J}^{i}\left(q^{2}\right)$ associated with even-chirality operators $S$ and located at $J=-1$ $-\frac{1}{2} \gamma_{S}+\gamma_{\psi}$. These poles arise from the factor $\Gamma\left(J+\frac{3}{2}+\frac{1}{2} p(\delta)\right)$ in the coefficient $a_{J}^{3}(\delta)$, Eq. (4.12). However, the residue $r^{i}$ at such a pole has only an $i=3$ component and $d^{23}\left[f^{(1)} \delta^{3 k}+f^{(2)} M^{3 k}\right] D^{k l}=0$, so there is no corresponding pole in $G(J)$. [The leading poles that do occur in $G(J)$ because of poles in the coefficients $a_{J}^{i}(\S)$ are located near $J=-2$.]

We now turn to the poles in $G(J)$ that arise from a divergence of the integral at large $q^{2}$. The terms in the wave function that are largest as $q^{2} \rightarrow \infty$ arise from the twist-two even-chirality operators $S=\bar{\psi} \gamma_{5}\left\{\gamma_{\mu} \partial_{\mu} \cdots \partial_{\mu}\right\} \psi$. These behave like

$$
\chi_{J}^{i}\left(q^{2}\right) \sim \sum_{\delta} a_{J}^{i}(\delta) C_{J}^{i}(\delta) q^{-3-\gamma} \delta+2 \gamma_{\psi}-J
$$

[see Eq. (4.11)]. Thus the large $-q^{2}$ part of the integral $G(J)$ behaves like

$$
\sum_{S \mathcal{S},} \int_{\Lambda}^{\infty} d q q^{4} q^{-3-\gamma_{S}}{ }^{+2 \gamma_{\psi}-J} q^{-3-\gamma_{\mathcal{S}}}{ }^{+2 \gamma_{\psi}-J-1} .
$$

Therefore $G(J)$ has "short distance" poles at

$$
J=-1+2 \gamma_{\psi}-\frac{1}{2} \gamma_{s}-\frac{1}{2} \gamma_{s^{\prime}},
$$

where $S$ and $S^{\prime}$ are any two of the twist-two operators $\bar{\psi} \gamma_{5}\left\{\gamma_{\mu} \partial_{\mu} \cdots \partial_{\mu}\right\} \psi$.

There are a few other mechanisms that could produce poles in $G(J)$, but do not. We have discussed divergences in the integration as $q^{2} \rightarrow \infty$. One might also imagine that the integration becomes divergent at some finite value $\tilde{q}^{2}$ of $q^{2}$ as $J$ takes on some particular value $\tilde{J}$. However, this would mean that $\chi_{J}^{i}\left(\tilde{q}^{2}\right)$ has a previously undiscovered singularity in the $J$ plane-a possibility that we have assumed does not occur. There remains the possibility of a divergence coming from the $q^{2} \rightarrow 0$ end of the integration. Inspection of the representation $(2.15)$ of $\chi_{J}^{i}\left(q^{2}\right)$ suggests that $\chi_{J}^{i}\left(q^{2}\right)$ behaves like $q^{L}$ as $q \rightarrow 0$, where $L$ equals the number of $\hat{q}^{\mu}$ 's in the basis function $G_{J}^{i}(L=J$ for $i=1,2$, $L=J-1$ for $i=3, L=J+1$ for $i=4$ ). This gives a leading small- $q$ behavior of the integrand in $G(J)$ of $q^{2 J+3}$ and thus a.: apparent pole at $J=-2$. Similar poles would appear to occur at other negative integer values of $J$. However, recall the Lorentz reflection symmetry properties of the wave functions, Eq. (2.23). These say, for instance, that $\chi_{-N}^{1}\left(q^{2}\right)=-\chi_{N-2}^{1}\left(q^{2}\right)$ for integer $N$. Since $\chi_{N-2}^{1}\left(q^{2}\right)$ has a $q^{2}$ dependence of $q^{N-2}$ instead of $q^{-N}$ as $q \rightarrow 0$, we conclude that the coefficients of the terms in $\chi_{J}^{1}\left(q^{2}\right)$ that are most singular as $q^{2} \rightarrow 0$ must have zeros at $J=-2,-3, \ldots$ These zeros (along with similar ones associated with $\chi_{J}^{i}$ for $\left.i=2,3,4\right)$ cancel the poles in $G(J)$ due to infrared divergences of the integral. Finally, the apparent poles caused by the factors $(J+2)^{-1}$ in the definition of $G(J)$ and $(J+1)^{-1}$ in the matrix $D_{J, J+1}^{j l}$ are canceled by zeros of the 
wave functions, as one can verify by using the Lorentz symmetry relations.

\section{Modifications for dressed quarks}

In the calculation just presented we have carefully analyzed the pion wave function, but we have simplified the quark inverse propagator and the quark-photon vertex in Fig. 2 by replacing them by $(i q q+m)$ and $\gamma_{\mu}$, respectively. If the full quark inverse propagator and vertex are used one would expect the results obtained above to be modified. One would also expect the modifications to be small in the limit of a small anomalous dimension $\gamma_{\psi}$ of the quark field, since in this limit far-off-shell quarks behave almost like bare quarks.

The pion form factor, including a dressed quark propagator and vertex, can be analyzed by using conformal invariance to determine the relevant behavior of the propagator and vertex and then using a modified version of the calculation presented above to extract the leading terms in $F\left(Q^{2}\right)$ as $Q^{2} \rightarrow \infty$. Since this analysis requires a large effort to produce a miniscule modification of the results, we content ourselves here with a statement of the modified results. A sketch of part of the required analysis is presented in the Appendix.

The quark-photon vertex is effectively modified by an extra factor (momentum) $)^{-2 \gamma} \psi$ when two or all three of its legs are far off shell. The quark inverse propagator is also modified by an extra factor (momentum $)^{-2 \gamma} \psi$ when it is far off shell. Consider first the "wave function" pole terms in $F\left(Q^{2}\right)$. These terms were $F_{S} \sim\left(Q^{2}\right)^{J}$ with $J$ given in Eq. (5.5) by $J=-1-\frac{1}{2} \gamma_{s}$, as determined by the behavior of the loop integrand when $(P+q)^{2} \rightarrow \infty$. These terms are now modified by an extra factor $\left(Q^{2}\right)^{-\gamma_{\psi}}$ from the vertex, giving

$$
F_{\delta}\left(Q^{2}\right)=\text { const } \times\left(Q^{2}\right)^{-1-\gamma_{S} / 2-\gamma_{\psi}} .
$$

Second, consider the "short-distance pole" terms $F_{S S}, \sim\left(Q^{2}\right)^{J}$ with $J$ given by Eq. (5.7): $J=-1+2 \gamma_{\psi}$ $-\frac{1}{2} \gamma_{s}-\frac{1}{2} \gamma_{s}$, as determined by the behavior of the loop integrand when all legs are far off shell. These terms are now modified by extra factors $\left(Q^{2}\right)^{-\gamma_{\psi}}$ from the vertex and $\left(Q^{2}\right)^{-\gamma_{\psi}}$ from the inverse propagator, giving

$$
F_{s s},\left(Q^{2}\right)=\text { const } \times\left(Q^{2}\right)^{-1-\gamma} s^{/ 2-\gamma} s^{\prime / 2} \text {. }
$$

\section{CONCLUSION}

We have analyzed the leading terms in the asymptotic expansion of the pion form factor and found terms of two types.

First, there are "short distance" terms connected with the large $-q^{2}$ behavior of the loop integration. One such term is associated with each pair of operators $S$ and $S^{\prime}$ chosen from among the twisttwo traceless symmetric operators $\psi \gamma_{5} \gamma_{\alpha} \psi$, $\bar{\psi} \gamma_{5}\left\{\gamma_{\alpha} \vec{\partial}_{\beta} \vec{\partial}_{\gamma}\right\} \psi, \ldots$ The corresponding term is

$$
E_{S S^{\prime}}\left(Q^{2}\right)=\text { const } \times\left(Q^{2}\right)^{-1-\gamma_{S} / 2-\gamma_{S}{ }^{\prime / 2}} \text {, }
$$

where $\gamma_{s}$ and $\gamma_{s}$, are the anomalous dimensions of $\delta$ and $\mathcal{S}^{\prime}$, respectively.

Second, there are "wave-function pole" terms associated with poles in the complex $\mathrm{O}(4) \mathrm{J}$ plane of the pion wave function. There is one such term for each of the twist-two operators enumerated above:

$$
F_{\delta}\left(Q^{2}\right)=\text { const } \times\left(Q^{2}\right)^{-1-\gamma_{S} / 2-\gamma} \psi .
$$

Here $\gamma_{\psi}$ is the anomalous dimension of the quark field $\psi(x)$ and, again, $\gamma_{\delta}$ is the anomalous dimension of $\delta$.

We can conclude that, as long as anomalous dimensions are small, the pion form factor behaves roughly like $\left(Q^{2}\right)^{-1}$ for large $Q^{2}$. A more detailed conclusion can be reached if we assume (1) that as one chooses operators $\&$ with higher and higher spin, $\gamma_{S} \rightarrow 2 \gamma_{\psi}{ }^{24}(2)$ that, as suggested by the ladder model, ${ }^{21} \gamma_{\delta}>2 \gamma_{\psi}$ for these operators $\delta$, and (3) that $\gamma_{\psi}>0 .^{25}$ On these assumptions the contributions from high-spin operators dominate the form factor. As long as the sum of these terms is absolutely convergent, one may conclude that the form factor falls off at least as fast as $\left(Q^{2}\right)^{-1-2 \gamma} \psi$ and that it does not fall off faster than $\left(Q^{2}\right)^{-1-2 \gamma_{\psi}-\epsilon}$, for any positive $\epsilon$.

These results can be compared with those of Callan and Gross ${ }^{3}$ and of Menotti. ${ }^{4}$ Callan and Gross obtained $F\left(Q^{2}\right) \sim\left(Q^{2}\right)^{-1-\gamma} S^{/ 2-\gamma_{\psi}}$, where $S$ was the leading odd-chirality operator, $\bar{\psi} \gamma_{5} \psi$. This and similar terms would indeed be present except that, as first pointed out by Menotti, the coefficient of these terms happens to vanish. [See Eq. (5.4).] Menotti then argued that the short-distance term corresponding to the operator $\bar{\psi}_{\gamma} \psi$ would dominate the form factor. Since this operator has twist three, the corresponding short-distance term is $F\left(Q^{2}\right) \sim\left(Q^{2}\right)^{-2}-\gamma_{S}$. Our analysis differs from that of Menotti by including the twist-two even-chirality operators $\bar{\psi} \gamma_{5} \gamma_{\mu} \psi, \ldots$, which, it turns out, provide the dominant terms in the form factor. ${ }^{26}$

The result obtained here also agrees approximately with the dimensional counting rule ${ }^{27}$ that the form factor of a bound state of $n$ quarks should behave like $\left(Q^{2}\right)^{1-n}$.

\section{ACKNOWLEDGMENTS}

We wish to thank C. Callan, D. Gross, L. Durand, A. Blaer, R. Blankenbecler, S. Brodsky, and N. Christ for very useful conversations. 


\section{APPENDIX: MODIFICATIONS FOR DRESSED QUARKS}

In the main text we made use of a bare quark propagator $(i q+m)$ and a bare quark-photon vertex $\gamma^{\mu}$. In this Appendix we will sketch, with some glaring lapses in rigor, the modifications that are necessary when one uses a dressed-quark inverse propagator and vertex.

The dressed inverse propagator has the simple form

$$
S_{F}^{-1}(q)=i \not q A\left(q^{2}\right)+B\left(q^{2}\right) .
$$

In order to compute the "short distance" terms in the asymptotic expansion of the pion form factor, we need to know the form of $S_{F}^{-1}(q)$ as $q^{2} \rightarrow \infty$. Conformal invariance ${ }^{25}$ at large $q^{2}$ dictates

$$
S_{F}^{-1}(q) \sim \text { const } \times i q\left(q^{2}\right)^{-\gamma_{\psi}}
$$

in this limit.

The dressed quark-photon vertex is not so simple. Let us call the (amputated) vertex $\Gamma^{\mu}\left(k^{\prime \nu}, k^{\nu}\right)$, where $k^{\prime \nu}$ is the momentum of the final quark and $k^{\nu}$ is the momentum of the initial quark. Lorentz invariance and parity allow us to write $\Gamma^{\mu}$ in terms of twelve form factors ${ }^{28}$ :

$$
\begin{aligned}
\Gamma^{\mu}\left(k^{\prime \nu}, k^{\nu}\right) & =\gamma^{\mu} f_{1}+k k^{\prime \mu} f_{2}+k^{\prime} k^{\mu} f_{3} \\
& +k k^{\mu} f_{4}+k^{\prime} k^{\prime \mu} f_{5}+k^{\prime} \gamma^{\mu} k f_{6} \\
& +k^{\mu} f_{7}+k^{\prime \mu} f_{8}+\sigma^{\mu \nu} k_{\nu} f_{9}+\sigma^{\mu \nu} k_{\nu}^{\prime} f_{10} \\
& +\sigma^{\alpha B} k_{\alpha}^{\prime} k_{B} k^{\mu} f_{11}+\sigma^{\alpha \beta} k_{\alpha}^{\prime} k_{B} k^{\prime \mu_{f}}{ }_{12} .
\end{aligned}
$$

The form factors are functions of $k^{\prime 2}, k^{2}$, and $Q^{2}$, where $Q^{\nu}=k^{\prime \nu}-k^{\nu}$.

As we will see, the only information about $\Gamma^{\mu}$ that is relevant for us is its dependence on $Q^{2}$ and $k^{2}$ when $Q^{2}$ and $k^{2}$ are large but $k^{\prime 2}$ is finite, its dependence on $Q^{2}$ and $k^{\prime 2}$ when these variables are large and $k^{2}$ is finite, and its dependence on all three variables when all three are large. We will assume that this information is correctly given by the conformally invariant vertex for two spinor fields and a conserved current, $\Gamma_{c}^{\mu}$. The conformal vertex has been given by Todorov. ${ }^{25}$ In momentum space it is

$$
\begin{aligned}
\Gamma_{c}^{\mu} & =k^{\prime}\left(k^{\prime 2}\right)^{-\gamma_{\psi}} \int \frac{d^{4} l}{(2 \pi)^{4}}\left(l^{2}\right)^{\gamma_{\psi-2}} \\
& =\left[C \frac{l+\not k^{\prime}}{\left(l+k^{\prime}\right)^{2}} \gamma^{\mu} \frac{l+\not k}{(l+k)^{2}}+D l \frac{l^{\mu}+k^{\mu}+l^{\mu}+k^{\prime \prime}}{(l+k)^{2}\left(l+k^{\prime}\right)^{2}}\right] \not\left(k^{2}\right)^{-\gamma_{\psi}},
\end{aligned}
$$

where $C$ and $D$ are constants not fixed by conformal invariance. [The derivation of Eq. (A4) involves the assumption that $\Gamma_{c}^{\mu}$ contains an odd number of $\gamma$ matrices. Terms in $\Gamma^{\mu}$ containing an even number of $\gamma$ matrices are smaller by a factor of (mass)/(momentum) in the large-momentum limit in perturbation theory.] One can extract the limiting behavior of the form factors $f_{n}$ from (A4) when needed by introducing Feynman parameters.

Let us consider now the contribution to the pion form factor from the term $\gamma^{\mu} f_{1}$ in $\Gamma^{\mu}$. We will treat this term as an example; the analysis of the other terms is similar in general method. We define the Mellin transform of $f_{1}$ :

$$
\tilde{f}_{1}\left(s^{\prime}, s, r\right)=\int_{0}^{\infty} d k^{\prime 2}\left(k^{\prime 2}\right)^{s^{\prime}-1} \int_{0}^{\infty} d k^{2}\left(k^{2}\right)^{s-1} \int_{0}^{\infty} d Q^{2}\left(Q^{2}\right)^{r-1} f_{1}\left(k^{\prime 2}, k^{2}, Q^{2}\right)
$$

The Mellin transform $\tilde{f}_{1}$ can be presumed initially to be analytic in $0<\operatorname{Re} s^{\prime}<\epsilon, 0<\operatorname{Re} s<\epsilon, 0<\operatorname{Re} r<\epsilon$ for some small $\epsilon$. We will discuss the singularity structure of $\tilde{f}_{1}$ in more detail shortly. The inversion of the Mellin transform is

$$
f_{1}\left(k^{\prime 2}, k^{2}, Q^{2}\right)=(2 \pi i)^{-3} \int_{a-i \infty}^{a+i \infty} d s^{\prime}\left(k^{\prime 2}\right)^{-s^{\prime}} \int_{a-i \infty}^{a+i \infty} d s\left(k^{2}\right)^{-s} \int_{a-i \infty}^{a+i \infty} d r\left(Q^{2}\right)^{-r} \tilde{f}_{1}\left(s^{\prime}, s, r\right)
$$

where $0<a<\epsilon$.

Let us substitute $\gamma^{\mu} f_{1}$ for $\gamma^{\mu}$ in the loop integral (3.4) for the pion form factor, with $f_{1}$ given by Eq. (A6) and the $s^{\prime}, s$, and $r$ integrations brought to the outside of the loop integration, to be done at the very end of the calculation. We also substitute the dressed-quark inverse propagator (A1) for $[i q+m]$. This gives

$$
F\left(Q^{2}\right)=(2 \pi i)^{-3} \int d s^{\prime} d s d r F\left(Q^{2}, s^{\prime}, s, r\right) \tilde{f}_{1}\left(s^{\prime}, s, r\right),
$$


where

$$
\begin{aligned}
\hat{F}\left(Q^{2}, s^{\prime}, s, r\right)=- & \frac{2 i}{Q^{2}+M^{2}}\left(Q^{2}\right)^{-r} \\
& \times \int_{0}^{\infty} q^{3} d q \int d \Omega \operatorname{Tr}\left\{\not P\left[(q+P)^{2}\right]^{-s} \chi\left(q^{\nu}+P^{\nu}, q^{\nu}\right)\left[i q A\left(q^{2}\right)+B\left(q^{2}\right)\right] \bar{\chi}\left(q^{\nu}, q^{\nu}+P^{\prime \nu}\right)\left[\left(q+P^{\prime}\right)^{2}\right]^{-s^{\prime}}\right\} .
\end{aligned}
$$

This expression for $\hat{F}\left(Q^{2} ; s^{\prime}, s, r\right)$ is nearly identical to the original expression (3.4) for $F\left(Q^{2}\right)$. The differences are the following:

(1) There is an overall factor $\left(Q^{2}\right)^{-r}$.

(2) The quark inverse propagator is modified, resulting in an extra factor $\left(q^{2}\right)^{-\gamma} \psi$ at large $q^{2}$.

(3) The wave function $\chi$ is modified to an effective wave function $\chi_{\text {eff }}=\left[(q+P)^{2}\right]^{-s} \chi$. From $\chi_{\text {eff }}$ one can form partial-wave amplitudes $\chi_{J}^{i}\left(q^{2}\right)_{\text {eff }}$ as before [see Eq. (2.7)]. The $J$-plane poles of $\chi_{J}^{i}\left(q^{2}\right)_{\text {eff }}$ tell how $\chi_{\text {eff }}$ falls off as $(q+P)^{2} \rightarrow \infty$. Therefore a pole of $\chi_{J}^{i}\left(q^{2}\right)$ that was located at $J=J(s, n)$ is now moved to $J=J(s, n)-s$. At fixed $J$ and large $q^{2}, \chi_{J}\left(q^{2}\right)$ falls off faster than $\chi_{J}\left(q^{2}\right)$ by a factor $\left(q^{2}\right)^{-s}$.

(4) The wave function $\bar{\chi}$ is similarly modified by a factor $\left[\left(q+P^{\prime}\right)^{2}\right]^{-s^{\prime}}$, so that its $J$-plane poles and power behavior at large $q^{2}$ are shifted by an a mount $s^{\prime}$.

If one now repeats the calculation in Secs. III and V, one finds that the asymptotic expansion of $\hat{F}\left(Q^{2} ; s^{\prime}, s, r\right)$ for large $Q^{2}$ is modified by small shifts in the powers of $Q^{2}$ that occur. The leading "short distance" terms in the expansion previously had the form

$$
F_{S S}^{(0)},\left(Q^{2}\right)=g\left(Q^{2}\right)^{-1+2 \gamma_{\psi}-\gamma_{S} / 2-\gamma_{S}, / 2}
$$

where $g$ is a constant [see Eq. (5.7)]. These terms now become

$$
\hat{F}_{S S},\left(Q^{2} ; s^{\prime}, s, r\right)=g\left(s^{\prime}, s, r\right)\left(Q^{2}\right)^{-1+2 \gamma_{\psi}-\gamma_{S} / 2-\gamma} S^{\prime / 2}\left(Q^{2}\right)^{-s^{\prime}-s-r-\gamma} \psi
$$

(The coefficient $g$ is now a function of $s^{\prime}, s$, and $r$, reflecting the dependence of the effective wave functions on $s$ and $s^{\prime}$ and on $J$, which has been set equal to $-1+\gamma_{\psi}-\gamma_{s} / 2-\gamma_{s}, / 2-s^{\prime}-s-r$.) The leading "wave-function pole" terms previously had the form

$$
F_{\text {SS }}^{(0)}\left(Q^{2}\right)=h\left(Q^{2}\right)^{-1-\gamma_{S} / 2}
$$

[see Eq. (5.5)]. These now become

$$
\hat{F}_{s}\left(Q^{2} ; s^{\prime}, s, r\right)=h\left(s^{\prime}, s, r\right)\left(Q^{2}\right)^{-1-\gamma} s^{/ 2}\left(Q^{2}\right)^{-r-s} \text {. }
$$

The pion form factor is given by Eq. (A 7) as an integral of the product of $\hat{F}_{s}\left(Q^{2} ; s^{\prime}, s, r\right)$ and the Mellin transform of the quark form factor. Let us consider the contribution from one of the "short distance" terms in $\hat{F}_{s}\left(Q^{2} ; s^{\prime}, s, r\right)$ given in Eq. (A9):

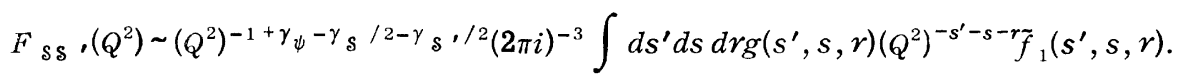

The $s^{\prime}, s$, and $r$ integrations initially run parallel to, and just to the right of, the imaginary $s^{\prime}, s$, and $r$ axes. The integral as it stands falls off at least as fast as $Q^{2}$ to the power $-\operatorname{Re} s^{\prime}-\operatorname{Re} s-\operatorname{Re} r$; this manifest rate of falloff can be increased by moving the integration contours to the right until a pole of the integrand is encountered.

The location of the leading pole in $f_{1}\left(s^{\prime} s, r\right)$ as $s^{\prime}+s+r$ increases tells how $f_{1}\left(k^{\prime 2}, k^{2}, Q^{2}\right)$ falls off as $k^{\prime 2}$, $k^{2}$, and $Q^{2}$ become large with $k^{2} / Q^{2}$ and $k^{2} / Q^{2}$ fixed. In this limit we expect $f_{1}$ to behave like the conformally invariant $f_{1}^{c}$ calculated from the conformally invariant vertex function $\Gamma_{c}^{\mu}$, Eq. (A4). In order to make the analysis as clear as possible, let us adopt a definite model for $f_{1}\left(k^{\prime 2}, k^{2}, Q^{2}\right)$ that approaches $f_{1}^{c}$ for large momenta, has good enough infrared behavior so that its Mellin transform exists, and is simple enough so that its Mellin transform is exactly calculable:

$$
f_{1}\left(k^{\prime 2}, k^{2}, Q^{2}\right)=\int_{0}^{1} d z d z_{1} d z_{2} d z_{3} \delta\left(z_{1}+z_{2}+z_{3}-1\right) \frac{z_{3}{ }^{2-\gamma_{\psi}}\left(k^{\prime 2}\right)^{1-\gamma_{\psi}}\left(k^{2}\right)^{1-\gamma_{\psi}}}{\left(z_{1} z_{3} k^{\prime 2}+z_{2} z_{3} k^{2}+z_{1} z_{2} Q^{2}+z_{1} z_{2} \Lambda^{2}\right)^{2-\gamma_{\psi}}}
$$


This model $f_{1}$ is the same as the conformally invariant function $f_{1}^{c}$ except for the term $z_{1} z_{2} \Lambda^{2}$ in the denominator. This term improves the infrared behavior of the model $f_{1}$ by making it finite at $k^{\prime 2}=k^{2}$ $=Q^{2}=0$, as one would expect for the true form factor $f_{1}$.

The Mellin transform of the model $f_{1}$ is

$$
\begin{aligned}
\tilde{f}_{1}\left(s^{\prime}, s, r\right)= & \Gamma\left(\gamma_{\psi}-s^{\prime}-s-r\right) \Gamma\left(s^{\prime}\right) \Gamma(s) \Gamma(r)\left(\Lambda^{2}\right)^{s^{\prime}+s+r-\gamma_{\psi}} \\
& \times \Gamma\left(1-\gamma_{\psi}+s^{\prime}\right) \Gamma\left(1-\gamma_{\psi}+s\right) \Gamma\left(1+\gamma_{\psi}-s-s^{\prime}\right) \\
& \times \Gamma\left(2-\gamma_{\psi}\right)^{-1} \Gamma\left(1+\gamma_{\psi}\right)^{-1} .
\end{aligned}
$$

We see that $\tilde{f}_{1}$ is analytic inside a tetrahedron in $\left(\operatorname{Re} s^{\prime}, \operatorname{Re} s, \operatorname{Re} r\right)$ space with sides at $\operatorname{Re} s^{\prime}=0$, $\operatorname{Re} s=0, \operatorname{Re} r=0$, and $\operatorname{Re} s^{\prime}+\operatorname{Re} s+\operatorname{Re} r=\gamma_{\psi}$ as indicated in Fig. 3.

Returning now to Eq. (A11), we move the integration contours to the largest value of $\operatorname{Re} s^{\prime}+\operatorname{Re} s$ $+\operatorname{Re} r$ attainable before encountering a singularity, that is, to $\operatorname{Re} s^{\prime}+\operatorname{Re} s+\operatorname{Re} r \simeq \gamma_{\psi}$. We then move, say, the $r$ integral past the pole at $s+s^{\prime}+r=\gamma_{\psi}$, picking up a residue term

$$
F_{\delta s} \cdot\left(Q^{2}\right)=\text { const } \times\left(Q^{2}\right)^{-1-\gamma} \delta^{/ 2-\gamma_{S}}, / 2
$$

and leaving a background integral that manifestly falls off faster then the residue term as $Q^{2} \rightarrow \infty$.

[We have neglected mentioning the singularities of the factor $g\left(s^{\prime}, s, r\right)$ in Eq. (A11). This factor can be expected to have a pole at $s^{\prime}=\gamma_{\psi}-\gamma_{S}, / 2$, since at this value of $s^{\prime}$ the "short distance" pole of the effective function $G(J)$, Eq. (3.11), at $J=-1$ $+\gamma_{\psi}-\gamma_{s} / 2-\gamma_{s}, / 2-s^{\prime}-s-r$ and the "wave function" pole at $J=-1-\gamma_{s} / 2-s-r$ coincide. We can simply choose not to move the $s^{\prime}$ contour past this pole.]

The contribution to $F\left(Q^{2}\right)$ from one of the "wavefunction pole" terms, Eq. (A10), in $\hat{F}\left(Q^{2} ; s^{\prime}, s, r\right)$ can be analyzed in a similar manner. We begin with

$$
\begin{aligned}
F_{S}\left(Q^{2}\right)= & \left(Q^{2}\right)^{-1-\gamma_{S} / 2}(2 \pi i)^{-3} \\
& \times \int d s^{\prime} d s d r h\left(s^{\prime}, s, r\right)\left(Q^{2}\right)^{-r-s_{f_{1}}}\left(s^{\prime}, s, r\right) .
\end{aligned}
$$

Here we want to increase $\operatorname{Re} s+\operatorname{Re} r$ as much as possible, moving near the edge of the tetrahedron in Fig. 3. If we then move, say, the $r$ contour

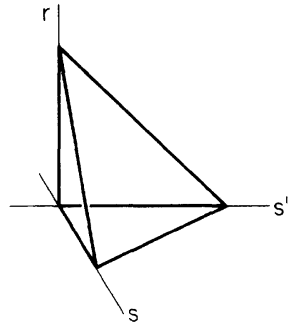

FIG. 3. Region of analyticity of the Mellin transform of the quark form factor.

past the pole of $\tilde{f}_{1}$ at $s^{\prime}+s+r=\gamma_{\psi}$ we obtain a background integral plus a dominant residue term:

$$
\begin{aligned}
& -\left(Q^{2}\right)^{-1-\gamma} s^{\prime 2}(2 \pi i)^{-2} \\
& \quad \times \int d s^{\prime} d \operatorname{sh}\left(s^{\prime}, s, \gamma_{\psi}-s^{\prime}-s\right)\left(Q^{2}\right)^{-\gamma_{\psi}+s^{\prime}} \\
& \quad \times \underset{r=\gamma_{\psi}-s^{\prime}-s^{\prime}}{\operatorname{Res}_{1}} \tilde{f}_{1}\left(s^{\prime}, s, r\right) .
\end{aligned}
$$

(This residue term can be thought of as represented by a point in Fig. 3 on the surface $\operatorname{Re} s^{\prime}+\operatorname{Re} s$ $+\operatorname{Re} r=\gamma_{\psi}$.) We can now increase the manifest rate of falloff of the residue term by moving the $s^{\prime}$ contour to the left, past the pole of $\operatorname{Res} \tilde{f}$ at $s^{\prime}=0$. This gives a background integral plus a double residue term:

$$
\begin{aligned}
& \left(Q^{2}\right)^{-1-\gamma_{S} / 2}\left(Q^{2}\right)^{-\gamma_{\psi}} \frac{1}{2 \pi i} \\
& \quad \times \int d \operatorname{sh}\left(0, s, \gamma_{\psi}-s\right) \underset{s^{\prime}=0}{\operatorname{Res}} \operatorname{Res}_{r=\gamma_{\psi}-s^{\prime}-s} \tilde{f}_{1}\left(s^{\prime}, s, r\right) .
\end{aligned}
$$

The background integrals fall off faster than the double residue term, so we have as $Q^{2} \rightarrow \infty$

$$
F_{\delta}\left(Q^{2}\right)=\text { const } \times\left(Q^{2}\right)^{-1-\gamma_{S} / 2-\gamma_{\psi}} .
$$

This completes the analysis of the contribution of the quark form factor $f_{1}$ to the pion form factor. The analysis of the other form factors is similar in spirit, although one must do some additional trace algebra in order to handle the more complicated spinor structure that accompanies these other form factors. Analysis of the other form factors leaves the results Eq. (A14) and Eq. (A16) unchanged (including the cancellation of the wavefunction pole term from the odd-chirality operators).

\footnotetext{
*This research was supported in part by the U. S. Energy Research and Development Administration and in part by the National Science Foundation under Grant No. MPS 75-22514.

${ }^{1} \mathrm{~J}$. S. Ball and F. Zachariasen, Phys. Rev. 170, 1541
}

(1968); D. Amati, L. Caneschi, and R. Jengo, Nuovo Cimento 58, 783 (1968); D. Amati, R. Jengo, H. R. Rubenstein, G. Veneziano, and M. A. Virasaro, Phys. Lett. 27B, 38 (1968).

${ }^{2}$ M. Ciafaloni and P. Menotti, Phys. Rev. 173, 1575 (1968). 
${ }^{3}$ C. G. Callan and D. J. Gross, Phys. Rev. D 11,2905 (1975).

${ }^{4}$ P. Menotti, Phys. Rev. D 13, 1778 (1976).

${ }^{5}$ M. L. Goldberger, A. H. Guth, and D. E. Soper (unpublished).

${ }^{6}$ See, for example, Handbook of Mathematical Functions, edited by M. Abramowitz and I. Stegun (National Bureau of Standards, Washington, 1964), Chapter 22.

${ }^{7}$ A. H. Guth and D. E. Soper, Phys. Rev. D $\underline{12}, 1143$ (1975).

${ }^{8}$ The wave function obeys $\chi\left(k_{1}, k_{2}\right) *=T \gamma^{4} \chi\left(k_{1}{ }^{*}, k_{2}{ }^{*}\right) \gamma^{4} T^{-1}$, as one can verify by combining Eq. (2.5) with the relation $\bar{\chi}\left(k_{1}, k_{2}\right)=-\gamma^{4} \chi\left(k_{2}{ }^{*}, k_{1} * \gamma^{4}\right.$ obtained by analytic continuation. The $G_{j}^{i}$ have been chosen to obey $G_{j}^{i}(q, P)^{*}=+T \gamma^{4} G_{j}^{i}\left(q^{*}, P^{*}\right) \gamma^{4} T^{-1}$. Thus the $\chi_{j}^{i}$ obey $\chi_{j}^{i}\left(q^{2}, P^{2}\right)^{*}=\chi_{j}^{i}\left(q^{2 *}, P^{2 *}\right)$ and are therefore real at points of analyticity on the real $q^{2}, P^{2}$ axes.

${ }^{9}$ See, for example, M. L. Goldberger and K. M. Watson, Collision Theory (Wiley, New York, 1964), pp. 634-639 and especially pp. $648-651$, where the quantity $-\zeta$ is our $q^{2}>0$.

${ }^{10}$ Such a term would have no discontinuity when $(q+P)^{2}$ $=-\rho^{2}$, so it would not contribute to $F_{D}$ in the dispersion relation for $F$ and thus would not contribute a pole in $g_{J}$ at $J=-N$. However, the formalism would remind us that the term existed by telling us at a certain point to look for poles of $\left(g_{J}+g_{-J-2}\right) / \sin \pi J$.

${ }^{11}$ G. C. Wick, Phys. Rev. 96, 1124 (1954). See also Ref. 7 for a more detailed discussion of the present notation. ${ }^{12}$ See, for example, Refs. 6 and 7.

${ }^{13}$ The identities in Appendix B of Ref. 1 are useful for carrying out the integrations.

${ }^{14}$ Write $\left(M^{2}\right)^{J-2} G(J-2) \equiv F(J) \equiv \int d q f(J, q)$. The integral over $q$ converges for large $\operatorname{Re} J$, and $F(J)$ is defined elsewhere by analytic continuation. The reflection properties (2.23) imply directly that $f(N, q)+f(-N, q)$ $=0$. The same statement for $F(J)$ follows by analytically continuing the expression

$F(N+z)+F(-N+z)$

$$
=z \int d q\{[f(N+z, q)+f(-N+z, q)] / z\}
$$

from large $\operatorname{Re} z$, where the integral is well defined, to $z=0$. The expression vanishes at $z=0$, and our result is proven, unless the continuation of the integral has a pole at $z=0$. Since the integrand is analytic at $z=0$, a pole will develop only by the same mechanisms which cause poles in $G(J)$, discussed in Sec. V. We assume that no such poles occur at integers.

${ }^{15} \mathrm{~K}$. Wilson, Phys. Rev. 179, 1499 (1969).

${ }^{16} \mathrm{G}$. Parisi, Phys. Lett. $\overrightarrow{39 B}, 643$ (1972). See also Ref. 3.

${ }^{17}$ C. G. Callan, S. Coleman, and R. Jackiw, Ann. Phys.
(N.Y.) $59,42(1970)$.

${ }^{18}$ C. G. Callan, Phys. Rev. D 2, 1541 (1970); K. Symanzik, Commun. Math. Phys. $\underline{18}, 227$ (1970); 23,49 (1971).

${ }^{19}$ S. Ferrara, R. Gatto, and A. F. Grillo, Nucl. Phys. B34, 349 (1971).

${ }^{20} \mathrm{~A}$. H. Guth and D. E. Soper (unpublished).

${ }^{21}$ The ladder-model results for the dimensions of various operators are obtained by comparing the ladder-model results for the pion wave function with the corresponding results from the operator-product expansion. See Ref. 5.

${ }^{22}$ One might worry that we have been too careless in extrapolating from integer values of $J$ into the complex $J$ plane. The recursion relations (4.5) and (4.6) can be solved in closed form for integer $J$ in terms of $\Gamma$ functions. Using the standard definition of the $\Gamma$ function for complex $J$, one discovers that the product $a_{j}^{i}(\mathcal{S}) C_{j}^{i}(S)$ has the poles discussed in the text, and behaves as a power of $J$ as $|J| \rightarrow \infty$. By Carlson's theor$\mathrm{em}$, there is only one continuation which is so well behaved.

${ }^{23}$ The matrix $\tilde{D}_{J, J+1}^{j l}=\left[f^{(1)} \delta^{j k}+q f^{(2)} M_{J}^{j k}\right] D_{J, J+1}^{k l}$ is essentially the same as $D_{J, J+1}^{j l}(m / q)$ :

$$
\tilde{D}=f^{(1)} D(m / q)+m f^{(2)} D(-q / m) .
$$

This is because multiplication of the quark inverse propagator $[i q+m]$ by $\left[f^{(1)}+i d f^{(2)}\right]$ produces no new $\gamma$-matrix structure on the quark line.

${ }^{24}$ C. J. Callan and D. J. Gross, Phys. Rev. D $\underline{8}, 4383$ (1973).

${ }^{25}$ I. T. Todorov, in Strong Interaction Physics, edited by W. Ruhl and A. Vancura (Springer, Berlin, 1973). ${ }^{26}$ Polyakov has argued from a different point of view that the twist-two operators, used as pion interpolating fields, dominate $F\left(Q^{2}\right)$ as $Q^{2} \rightarrow \infty$. However, he appears to neglect the "wave-function pole" or "conformal anomaly" terms, which vanish only for the twist-three operators. See A. M. Polyakov, in Proceedings of the 1975 International Symposium on Lepton and Photon Interactions at High Energies, edited by W. T. Kirk (SLAC, Stanford, California, 1976).

${ }^{27}$ S. J. Brodsky and G. R. Farrar, Phys. Rev. Lett. 31, 1153 (1973); Phys. Rev. D 11, 1309 (1975); V. Matveev, R. Muradyan, and A. Tavkhelidze, Lett. Nuovo Cimento $\underline{7}, 719$ (1973); R. Blankenbecler, S. Brodsky, and J. Gunion, Phys. Rev. D $\underline{8}, 187$ (1973). See also D. Sivers, S. J. Brodsky, and R. Blankenbecler, Phys. Rep. 23,1 (1976).

${ }^{28}$ There are relations among the form factors due to charge-conjugation invariance and current conservation, but these relations are not useful to us. 